molecule at special points, where there is a particularly strong stray field, say, for example, where the amino and acid groups of the amino acids come together. It may be that later work will throw light on this point.

URBANA, rirh.

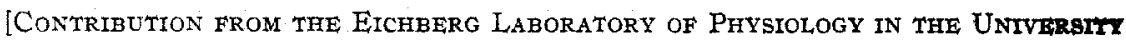
of CINCINNat1.]

\title{
ON THE SWELLING OF GELATIN IN POLYBASIC ACIDS AND THEIR SALTS. ${ }^{1}$
}

By Martin H. Fischer and Marian O. Hooker.

Received October 31, 1917.

I. Introductory.

It is now safe to conclude that the amount of water absorbed by any living cell, organ or organism, is essentially dependent upon its content of hydrophilic colloids and the state of these. As proof for this may be cited the complete analogy which exists between the laws governing the absorption of water by protoplasm and those governing the same phenomenon as observed in various simple colloids-more particularly the proteins-under physiological and pathological conditions." On such a colloid-chemical basis it is now easy to account for the fairly constant amount of water held by living cells and tissues normally and so to explain, for example, their so-called normal "turgor." But under pathological conditions both in plants and in animals this normal degree of hydration may be much increased, when we have before us the "excessive turgor" characteristic of various plant diseases, or the edema of the animal pathologists as this involves individual cells, organs, or the body as a whole. Edema may, in other words, be defined as a state of excessive hydration of the body colloids, while as "causes" of the edema may be cited substances or conditions which under the circumstances prevailing in living protoplasm are capable of increasing its water holding powers to beyond the normal limits.

In connection with this problem of the "causes" of edema a study of the conditions which under laboratory circumstances increase the hydration capacity of different colloids has again yielded valuable hints as to the nature of those which are active in living protoplasm. Among the substances thus active so far as the proteins are concerned, acids occupy a first place; important, too, are the amines; while effective in this regard,

1 A preliminary statement of the results detailed in this paper appeared in Science, 46,189 (1917).

2 Martin H. Fischer, "Physiology of Alimentation," 250, 268, New York, I917; Am. J. Physiol,, 20, 339 (1907); Pflüger's Arch., 124, 69 (1908); for a running account and detailed references, see Fischer: "Edema and Nephritis," and Ed., New York. 1915 . 
though not, perhaps, of much physiological or pathological importance, are pyridine and urea. In other words, we may expect an edema to result whenever in any living tissue there occurs an abnormal production or accumulation of these substances.

There has been much that is foolish written against these simple conclusions. Insofar as many of these criticisms rest upon a misreading or an actual violation of what we have written we shall not trouble to correct them. It is the purpose of this paper to revert to that oft-repeated objection that an abnormal production or accumulation of acids in a cell, tissue or organ can not be a potent cause of edema because our tissues contain phosphates or other "buffer" salts which have the power of taking up considerable amounts of either acid or alkali without change in "hydrogen-ion acidity." 1 Aside from the facts often pointed out before that such buffer action is not unlimited in amount, that there is a vast difference between our constantly reiterated "acid content" of tissues and their hydrogen-ion concentration, that the physiological action of even pure acids nowhere parallels their electrolytic dissociation, and that even with extreme variations in acid content there may be little change in hydration capacity if various neutral salts are present, the data in this paper show clearly enough that from a given low point, even in the presence of such "buffer" salts, there is a progressive increase in water absorption by various proteins with every increase in the acid or alkali content of the mixture.

\section{Experimental Methods.}

Dried gelatin discs prepared in the accepted fashion ${ }^{2}$ served as the material upon which to test out the effects of different polybasic acids and their salts. For the polybasic acids we chose phosphoric and carbonic because of their importance in the animal body, and citric because of its great role in the plant economy. From the standardized solutions of these acids and of sodium hydroxide were then made the necessary primary, binary or ternary salts, by mixing the acid and alkali together in the theoretically necessary amounts.

The figures and tables are largely self-explanatory. The changes in weight (water absorption) of the gelatin discs at the end of different periods are calculated in terms of the original dry weight of the disc taken as unity.

\section{Experiments with Phosphate Mixtures.}

It was necessary, first, to determine the amounts of water absorbed by gelatin in different concentration of the mono-, di-, and trisodium phosphates and to discover how long a time is necessary before this water absorption is approximately complete. The results of three such experi-

1 See for example Max Koppel, Deut. Arch. Klin. Med., r12, 594 (1913); L. J. Henderson, W. W. Palmer and L. H. Newburgh, J. Pharm. Exp. Therap., 5, 449 (I9I4).

2 See Martin H. Fischer, "Edema and Nephritis," and Ed., 57, New York, 1915. 
ments are shown in the curves of Figs. 1,2 and 3 , as well as in Tables I, II and II, which contain the data from which the curves are drawn.

TABLE I.

Gelatin-Monosodium phosphate.

\begin{tabular}{|c|c|c|c|c|c|}
\hline 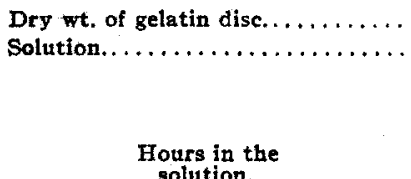 & $\begin{array}{c}0.311 \\
5 \mathrm{cc} . \\
1 M \\
\mathrm{NaH}_{4} \mathrm{PO}_{4} \\
+95 \mathrm{cc} \\
\mathrm{H}_{3} \mathrm{O}\end{array}$ & $\begin{array}{c}0.312 \\
15 \mathrm{cc} \\
1 \mathrm{M} \\
\mathrm{NaH}_{2} \mathrm{PO} \\
+85 \mathrm{cc} \\
\mathrm{H}_{2} \mathrm{O} \\
\text { Gain in part } \\
\end{array}$ & $\begin{array}{c}0.312 \\
25 \mathrm{cc} \\
1 \mathrm{M} \\
\mathrm{NaHzPO} \\
+75 \mathrm{cc} \\
\mathrm{H}_{8} \mathrm{O} \\
\mathrm{s}, \text { of one par }\end{array}$ & $\begin{array}{c}0.314 \\
50 \mathrm{cc} . \\
1 \mathrm{M} \\
\mathrm{NaH}_{2} \mathrm{PO}_{4} \\
+50 \mathrm{cc} \\
\mathrm{H}, \mathrm{O} \\
\text { rt of gelatin. }\end{array}$ & $\underset{\mathrm{H}_{2} \mathrm{O}}{100 \mathrm{cc}}$ \\
\hline 18 & $7 \cdot 31$ & $7 \cdot 75$ & 7.73 & 7.12 & $7 \cdot 30$ \\
\hline 24 & 7.71 & 8.25 & 8.16 & 7.55 & 7.60 \\
\hline 42 & 8.27 & 9.00 & 8.83 & 8.20 & 8.45 \\
\hline 48 & 8.50 & 9.20 & 9.12 & 8.38 & 8.90 \\
\hline 66 & 8.65 & 9.37 & 9.25 & 8.60 & 9.80 \\
\hline 89 & 9.03 & 9.87 & 9.73 & 9.00 & II.9I \\
\hline Curve designation. & I & II & III & IV & $\mathrm{H}_{2} \mathrm{O}$ \\
\hline
\end{tabular}

TABLE II.

Gelatin-Disodium Phosphate.

\begin{tabular}{|c|c|c|c|c|c|}
\hline \multirow{2}{*}{$\begin{array}{l}\text { Dry wt. of gelatin disc......... } \\
\text { Solution..................... } \\
\text { Hours in the } \\
\text { solution. } \\
\text { I } 8\end{array}$} & \multirow{2}{*}{$\begin{array}{c}0.317 \\
5 \mathrm{cc} \\
1 M \\
\mathrm{NazHPO} \\
+95 \mathrm{cc} \\
\mathrm{H} . \mathrm{O}\end{array}$} & $\begin{array}{c}0.317 \\
15 \mathrm{cc} . \\
1 \mathrm{M} \\
\text { Nay HPO } \\
+8.5 \mathrm{cc} . \\
\text { Hy } \\
\text { Gain in parts }\end{array}$ & $\begin{array}{l}0.319 \\
25 \mathrm{cc} . \\
1 M \\
\text { Naspo, } \\
+75 \mathrm{cc} \\
\text { His. } \\
\text { s, of one part }\end{array}$ & $\begin{array}{c}0.319 \\
50 \mathrm{cc} . \\
1 \mathrm{M} \\
\mathrm{NaHPO}_{4} \mathrm{HPO}_{4} \\
+50 \mathrm{cc} . \\
\text { HzO } \\
\text { tof gelatin. }\end{array}$ & $\begin{array}{c}0.315 \\
100 \mathrm{cc} \\
\mathrm{H}, \mathrm{O}\end{array}$ \\
\hline & & 8.57 & 7.80 & 5.60 & 6.84 \\
\hline 24 & 9.74 & 9.16 & 8.30 & 5.91 & $7 \cdot 4 \mathrm{I}$ \\
\hline 42 & 10.65 & 10.08 & 9.06 & 6.40 & 8.20 \\
\hline 48 & IO.9I & 10.32 & 9.30 & 6.56 & 8.60 \\
\hline 66 & II.I 6 & 10.70 & 9.63 & 6.74 & 10.43 \\
\hline 89 & II. 65 & 11.22 & 10.20 & 7.24 & 11.22 \\
\hline Curve designation. . & I & II & III & IV & $\mathrm{H}_{2} \mathrm{O}$ \\
\hline \multicolumn{6}{|c|}{$\begin{array}{c}\text { TABLE III. } \\
\text { Gelatin-Trisodium Phosphate. }\end{array}$} \\
\hline $\begin{array}{l}\text { ry wt. of gelatin disc......... } \\
\text { dlution... } \ldots \ldots \ldots \ldots \ldots \ldots\end{array}$ & $\begin{array}{c}0.321 \\
5 \mathrm{cc} \\
1 \mathrm{M} \\
\mathrm{Na}_{4} \mathrm{PO} \\
+95 \mathrm{cc} \\
\mathrm{H}_{2} \mathrm{O}\end{array}$ & $\begin{array}{c}0.322 \\
15 \mathrm{cc} . \\
1 \mathrm{M} \\
\mathrm{Na}_{4} \mathrm{PO}_{4} \\
+85 \mathrm{cc} . \\
\mathrm{H}_{1} \mathrm{O} \\
\text { Gain in parts. }\end{array}$ & $\begin{array}{c}0.322 \\
25 \mathrm{cc} . \\
1 M \\
\text { Napo. } \\
+75 \mathrm{cc} \\
\text { HaO } \\
\text { of one part }\end{array}$ & $\begin{array}{c}0.323 \\
50 \mathrm{cc} \\
1 \mathrm{M} \\
\mathrm{NaPO} \\
+50 \mathrm{cc} \\
\text { Hyo } \\
\text { of gelatin. }\end{array}$ & $\begin{array}{c}100 \mathrm{cc} \\
\mathrm{H}, \mathrm{O}\end{array}$ \\
\hline I 8 & 12.44 & 10.30 & 8.44 & 3.77 & $5 \cdot 32$ \\
\hline 24 & 13.97 & xI.67 & 9.62 & 4.06 & 6.52 \\
\hline 42 & $17 \cdot 3 I$ & 15.28 & I2.94 & 4.63 & $7 \cdot 75$ \\
\hline 48 & 18.26 & $16.6 \mathrm{I}$ & 14.10 & 4.83 & 8.20 \\
\hline 66 & 20.93 & $19.7 \mathrm{I}$ & 17.20 & 5.10 & $9.2 \mathrm{I}$ \\
\hline 89 & 22.46 & 22.22 & 21.80 & $5 \cdot 55$ & 10.72 \\
\hline lesigna & & II & III & IV & $\mathrm{H}_{2} \mathrm{O}$ \\
\hline
\end{tabular}

As evidenced in Fig. I, gelatin swells little more or little less in any solution of monosodium phosphate between the concentrations of 0.05 
and $0.5 M$ than it does in pure water. Fig. 2 shows that disodium phosphate in its lower concentrations tends to increase the swelling of gelatin. A maximal swelling is observed in the least concentrated solution of this salt. With increasing concentration the amount of swelling decreases, so that by the time the $0.5 M$ disodium phosphate solution is reached, the swelling of the gelatin is distinctly less than in pure water. A similar rule holds for like concentrations of trisodium phosphate, as shown in Fig. 3. Gelatin swells distinctly more in low concentrations of trisodium phosphate than in water, though

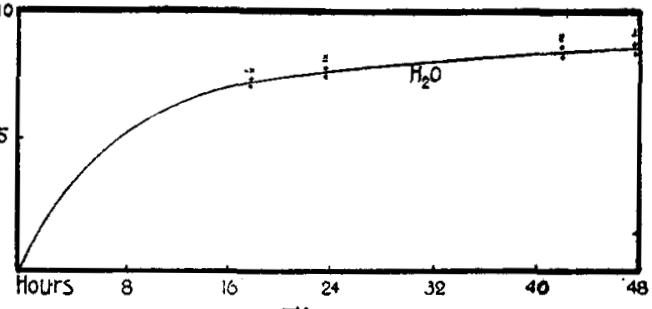

Fig. I.

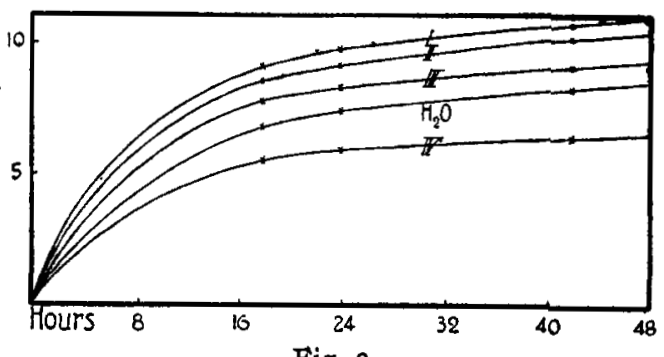

Fig. 2. phate solution at the end of 24 to 48 hours. We therefore settled upon such approximate periods for the next series of experiments in which is we sought to discover the amount of swelling shown by gelatin when immersed in so- 10 lutions varying from pure phosphoric acid, on the one hand, through mono-, di- and trisodium phosphate mixtures to pure sodium hydroxide on the other. It was our purpose in this experiment to see how the amount of swelling fared

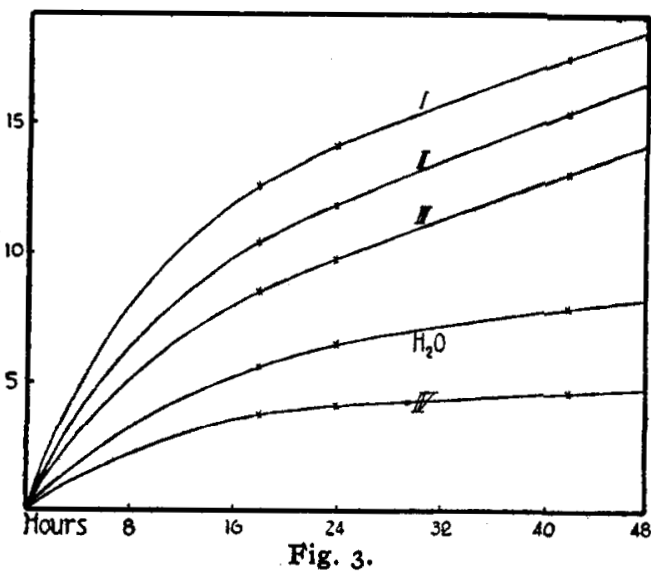
as more and more of the initially pure phosphoric acid was replaced by the mono-, di-, or trisodium salt. The result is shown in Table IV, and Fig. 4. The curves show the amounts of swelling at the end of 24 and of 48 hours. The low point in the curve indicative of least swelling is representative of the effects observed in the phosphate mixture numbered 6 in Table IV. 
As readily apparent, every increase in acid content to the left of this point is followed by increased swelling and the same is true for every increase in alkali content to the right of this point.

TABLE IV.

Gelatin-Phosphoric Acid to Phosphates, to Sodium Hydroxide.

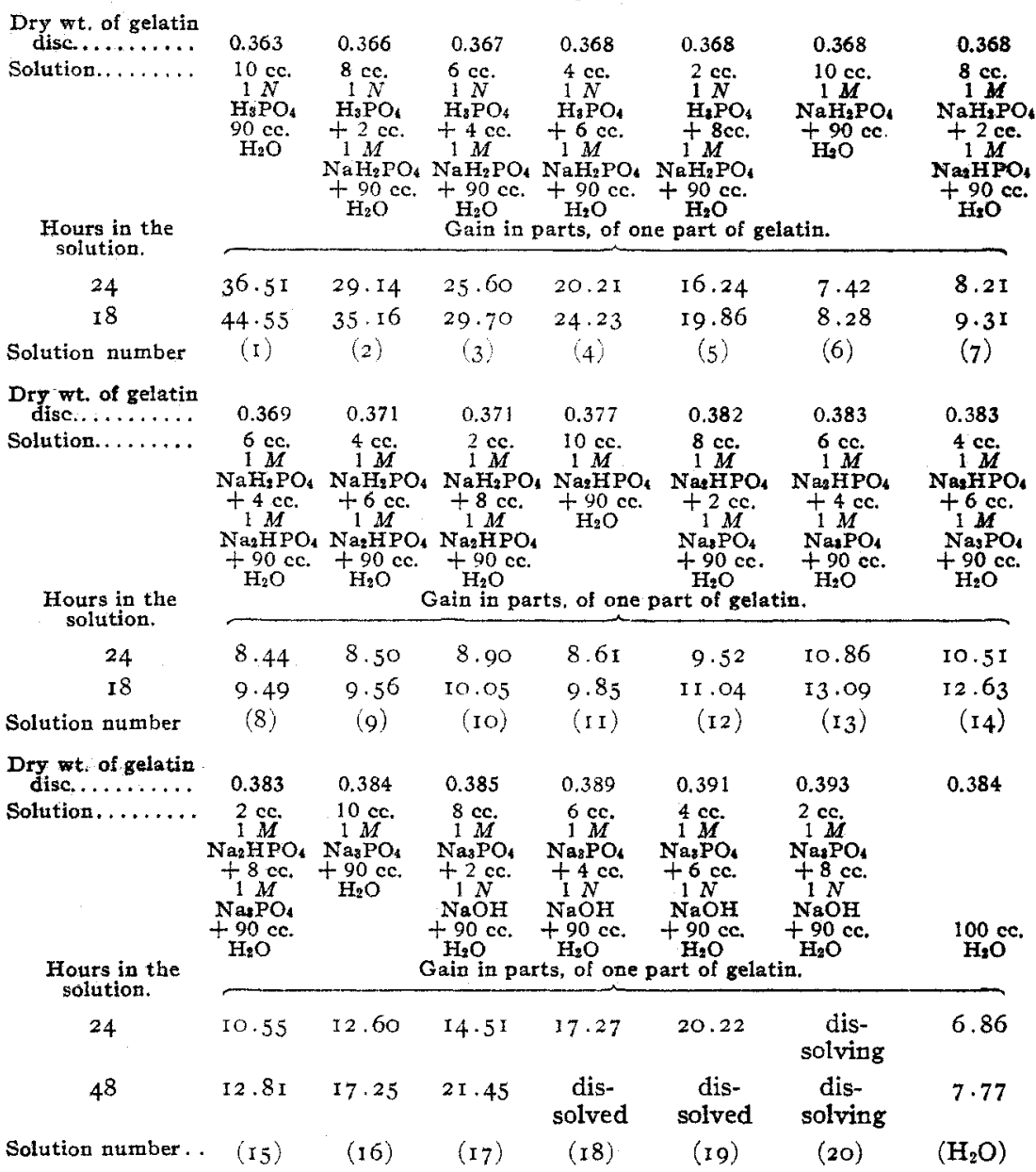

It is an interesting fact that the hydrogen-ion concentration of Solution 6 is about that indicated by the turning point of methyl red $\left(\mathrm{IO}^{-6} \mathrm{C}_{\mathrm{H}}\right.$ ). While we would not have it thought that the behavior of gelatin is at once to be paralleled with that of our body proteins, it is a fact that clinical observation indicates that urinary hydrogen-ion acidities approach an unsafe height when they begin to lie persistently above this point. ${ }^{1}$

i See Martin H. Fischer, Trans. Assoc. Am. Phys., 27, 630 (1912); "Edema and Nephritis," 2nd Ed., 635, New York, I9r5. 


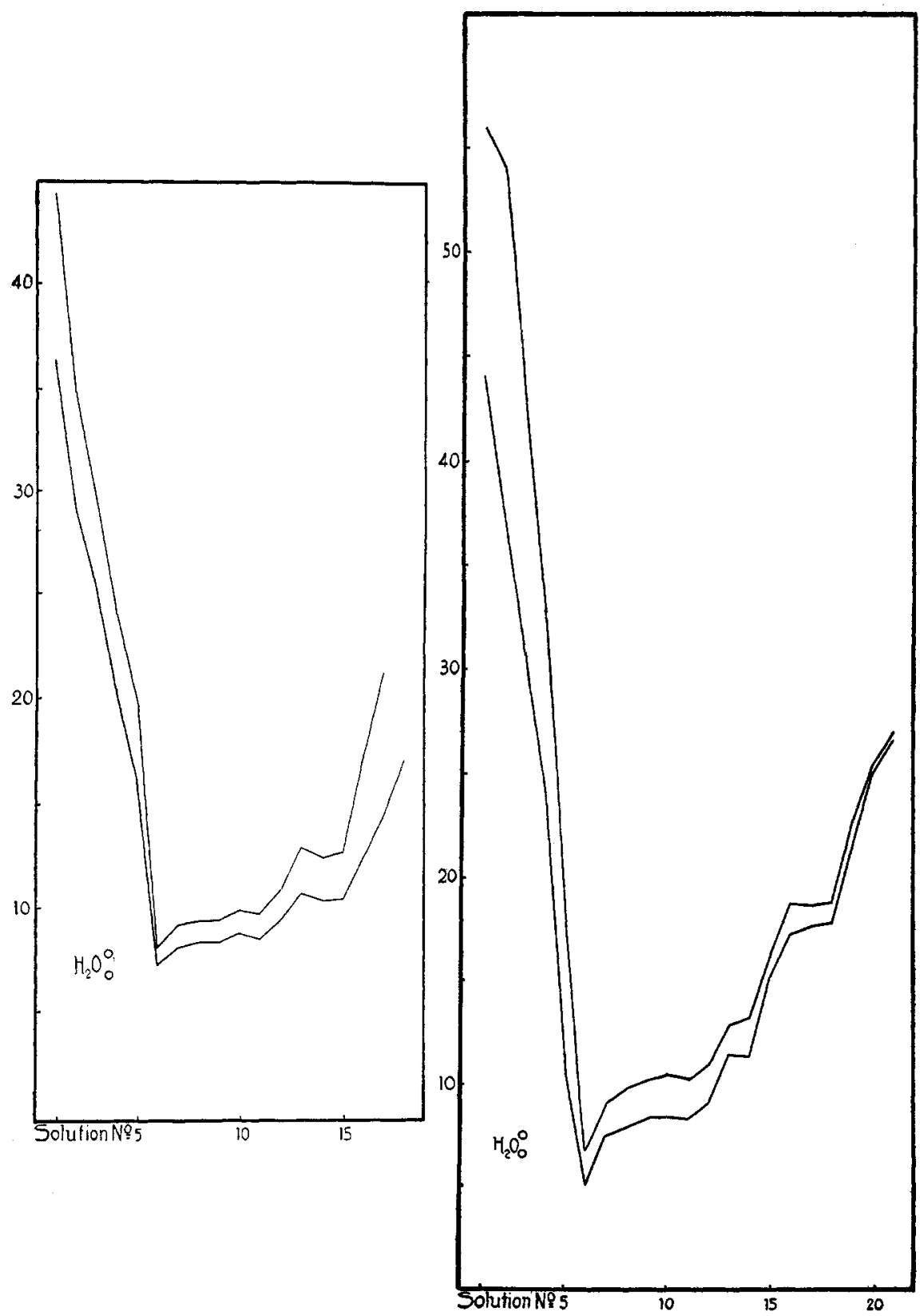

Fig. 4.

Fig. 5. 
Since the concentration of the phosphate mixtures employed in the experiments just described is relatively high ( $0.1 M$ ), we did a second series at a concentration approximating that of the phosphates in the human body. The results are shown in Fig. 5 and Table V. The general shapes of the two curves which again represent the amounts of swelling attained in the different solutions at the ends of 18 and 42 hours are identical with those shown in Fig. 4. It is of interest to point out that the low point in the swelling curves representative of the effects of this more dilute phosphate mixture falls below the amount of swelling attained in pure water. There is, however, a proportionally greater swelling as we

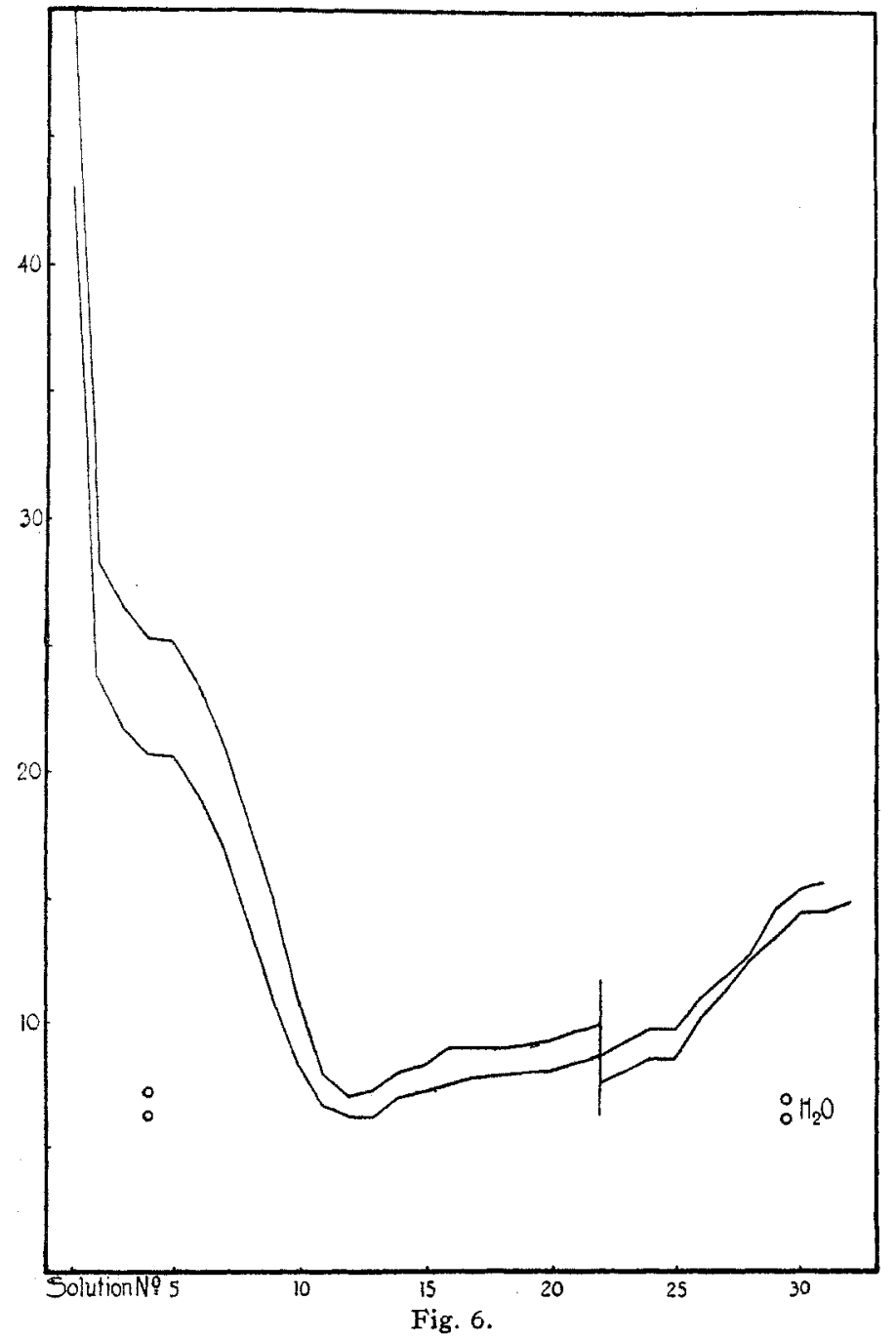


move from this minimal point either in the direction of an increased acid or increased alkali content of the solutions, even though the proportions in the various mixtures are identical with those used in the experiments of Table IV. The absolute amounts of swelling in the acid and alkaline extremes of these more dilute phosphate mixtures are distinctly higher than in the more concentrated phosphate series previously described.

In the experiments listed in Tables IV and V, the molar concentration of the phosphate is the same throughout. While the curves of Figs. 4 and 5 show that every increase in the acid or alkali content of the phosphate mixture on either side of a low point 20 is followed by an increased water absorption on the part of the immersed protein, it might be argued that such conditions do not obtain in the living organism where, it might be insisted, we begin with a definite concentration of a certain phosphate and then see an acid or an alkali added to this. That under such circumstances we also get a progressive increase in swelling as either the acid or alkali content of the

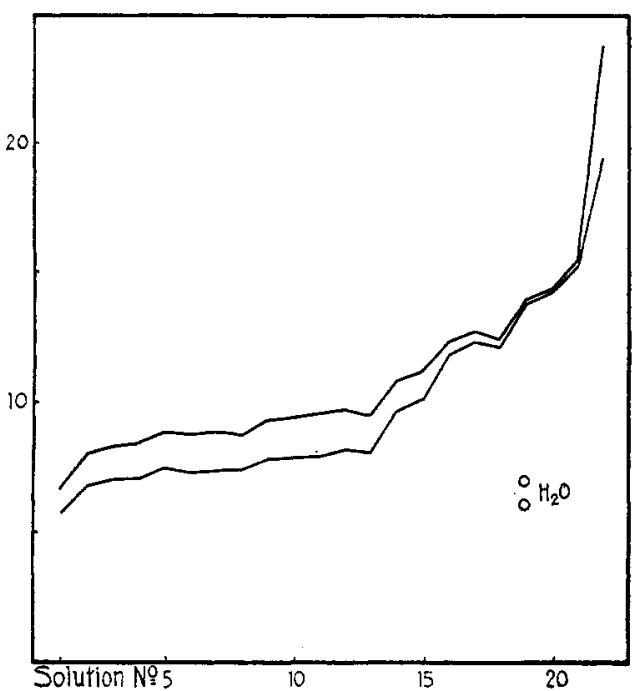

Fig. 7. mixture rises is shown in Figs. 6 and 7 and Tables VI and VII. The first of these figures and tables begins with a fixed concentration of disodium phosphate (o.or $M$ ), the second, with the same concentration of the monosodium salt.

TABLE V.

Gelatin-Phosphoric Acid to Phosphates, to Sodium Hydroxide.

\begin{tabular}{|c|c|c|c|c|c|c|c|}
\hline$r y$ & & & & & & & \\
\hline olution...... & $\begin{array}{c}1 \mathrm{cc} . \\
1 \mathrm{~N} \\
\mathrm{H}_{8} \mathrm{PO} \\
+99 \mathrm{cc} . \\
\mathrm{H}_{1} \mathrm{O}\end{array}$ & $\begin{array}{c}0.8 \mathrm{cc} \\
1 \mathrm{~N} \\
\mathrm{H}_{3} \mathrm{PO} \\
+0.2 \mathrm{cc} \\
1 \mathrm{M} \\
\mathrm{NaH}_{2} \mathrm{PO}_{4} \\
+99 \mathrm{cc} . \\
\mathrm{H}, \mathrm{O}\end{array}$ & $\begin{array}{c}0.6 \mathrm{cc} \\
1 N \\
\mathrm{H}_{3} \mathrm{PO} \\
+0.4 \mathrm{cc} . \\
1 \mathrm{M} \\
\mathrm{NaH}_{2} \mathrm{PO}_{4} \\
+99 \mathrm{cc} . \\
\mathrm{H}_{9} \mathrm{O}\end{array}$ & $\begin{array}{c}0.4 \mathrm{cc} . \\
1 \mathrm{~N} \\
\mathrm{H}, \mathrm{PO} \\
+0.6 \mathrm{cc} \\
11 M \\
\mathrm{NaH}_{3} \mathrm{PO}_{4} \\
+99 \mathrm{cc} \\
\mathrm{H}, \mathrm{O}\end{array}$ & $\begin{array}{c}0.2 \mathrm{cc} . \\
1 \mathrm{~N} \\
\mathrm{H}_{2} \mathrm{PO}_{4} \\
+0.8 \mathrm{cc} . \\
1 \mathrm{M} \\
\mathrm{NaH}_{8} \mathrm{PO}_{4} \\
+99 \mathrm{cc} \\
\mathrm{H}_{2} \mathrm{O}\end{array}$ & $\begin{array}{c}1 \mathrm{cc} . \\
1 M \\
\mathrm{NaF} \\
+99 \mathrm{cc} \\
+99 . \\
\mathrm{H}_{2} \mathrm{O}\end{array}$ & $\begin{array}{c}0.8 \mathrm{cc} . \\
1 \mathrm{M} \\
\mathrm{NaH} \mathrm{HO}_{4} \\
+0.2 \mathrm{cc} . \\
1 \mathrm{M} \\
\mathrm{Na} \mathrm{MPO} \\
+99 \mathrm{cc} . \\
\mathrm{H}, \mathrm{O}\end{array}$ \\
\hline & & & 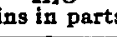 & $2+20$ & & & \\
\hline I 8 & 44.00 & 37.60 & 31.02 & 22.94 & I0. 49 & & 7.65 \\
\hline 42 & $55 \cdot 97$ & $53 \cdot 97$ & 42.84 & $32 \cdot 73$ & $17 \cdot 54$ & 6.88 & 9.14 \\
\hline olution numb & (I) & (2) & (3) & (4) & (5) & (6) & $(7)$ \\
\hline
\end{tabular}




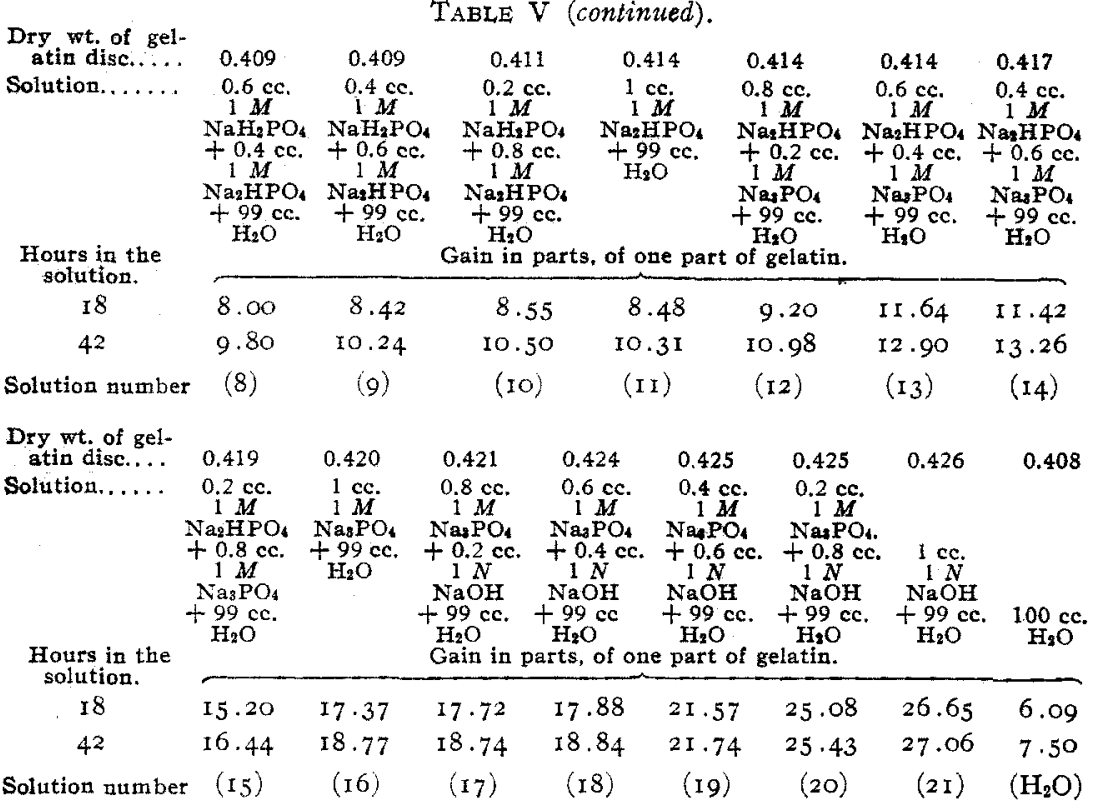

TABLE VI.

Gelatin-Disodium Phosphate + Phosphoric Acid or Sodium Hydroxide.

Dry wt. of gel-

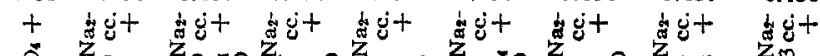

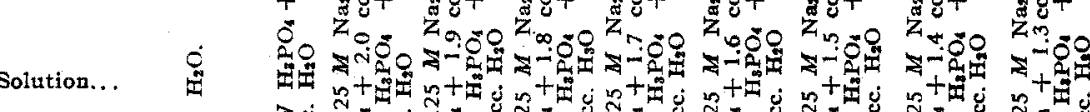

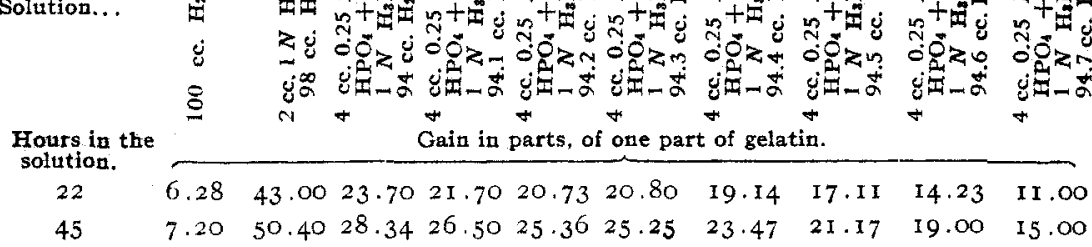
Solution No. $\left(\mathrm{H}_{2} \mathrm{O}\right)$
(I)
(2)
(3)
(4)
(5)
(6)
(7)
(8)

(9)

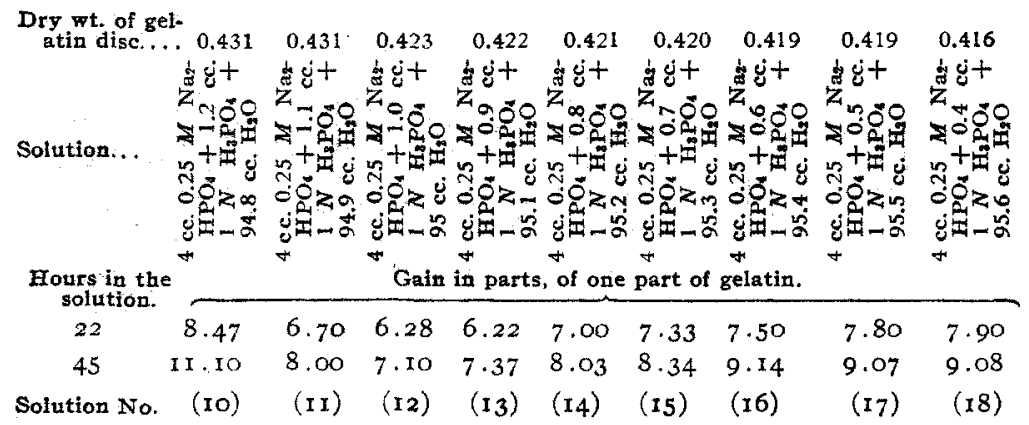


TABLE VI (continued).

Dry wt. of gel-

$\begin{array}{lllllllll}\text { atia disc.... } & 0.415 & 0.414 & 0.413 & 0.413 & 0.405 & 0.406 & 0.406 & 0.407\end{array}$

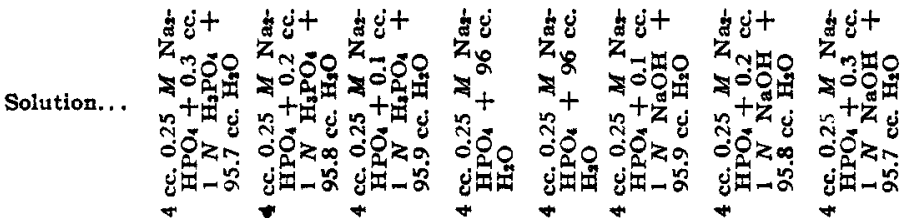

Hours in the solution.

22

Gain in parts, of one part of gelatin.

45

$\begin{array}{lllllllll}8.00 & 8.16 & 8.45 & 8.68 & 7.63 & 8.03 & 8.57 & 8.60\end{array}$

$\begin{array}{llllllll}9.10 & 9.30 & 9.70 & 10.06 & 8.72 & 9.20 & 9.82 & 9.70\end{array}$

$\begin{array}{lllllllll}\text { Solution No. } & (19) & (20) & (2 I) & (22) & (23) & (24)^{\prime} & \text { (25) } & \text { (26) }\end{array}$

Dry wt. of gel-

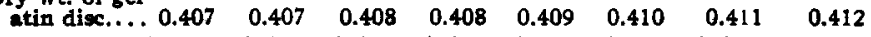

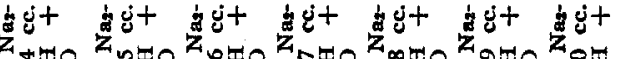

Solution...

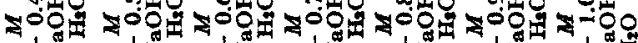
坛 今ึ

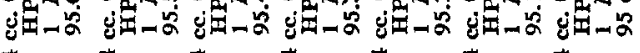

8

Fours in the solution.

Gain in parts, of one part of gelatin.

$\begin{array}{lllllllll}22 & \text { IO.29 } & \text { II } .21 & \text { I2.65 } & \text { I3.4I } & \text { I4.40 } & \text { I4.57 } & \text { I4.87 } & 6.04 \\ 45 & \text { II.O5 } & \text { II } .80 & \text { I2.72 } & \text { I3.65 } & \text { I5.40 } & \text { I5.70 } & \text { I4.8I } & 6.82\end{array}$

Solution No. (27) (28) (29) (30) (3I) (32) $\quad(33) \quad\left(\mathrm{H}_{2} \mathrm{O}\right)$

TABLE VII.

Gelatin-Monosodium Phosphate, with Increasing Amounts of Sodium Hydroxide.

Dry wt. of gel-

$\begin{array}{rllllllll}\text { atin disc.... } & 0.388 & 0.390 & 0.391 & 0.392 & 0.392 & 0.393 & 0.393 & 0.393\end{array}$

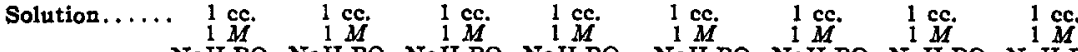

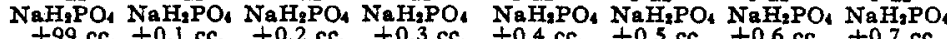
$+99 \mathrm{cc}+0.1 \mathrm{cc}$ + $0.2 \mathrm{cc}+0.3 \mathrm{cc}+0.4 \mathrm{cc}$. $0.5 \mathrm{cc}+0.6 \mathrm{cc}$. $+0.7 \mathrm{cc}$.

$\mathrm{NaOH} \mathrm{NaOH} \mathrm{NaOH} \mathrm{NaOH} \mathrm{NaOH} \mathrm{NaOH} N \mathrm{NaOH}$ $+98.9 \mathrm{cc}$. $+98.8 \mathrm{cc}+98.7 \mathrm{cc}$. $+98.6 \mathrm{cc}$. $+98.5 \mathrm{cc}$. $+98.4 \mathrm{cc}$. $+98.3 \mathrm{cc}$.

Hours in

the solution.

20 $\underset{\mathrm{Gain}}{\mathrm{H}_{2} \mathrm{O}} \begin{gathered}\mathrm{H}_{2} \mathrm{O} \\ \mathrm{H}\end{gathered}$

44

$5 \cdot 90$

$\mathrm{H}_{2} \mathrm{O}$

Solution No...

6.90

7 . I I

7 . I I

7.65

$7 \cdot 47$

$\mathrm{H}_{2} \mathrm{O}$

$\mathrm{H}, \mathrm{O}$

Dry wt, of gel-

atin disc....

Solution.....

(I)

(2)

(3.)

(4)

(5)

(6)

$7 \cdot 50$

$7 \cdot 53$

$0.394 \quad 0.395$

0.395

0.397

0.398

0.400

(7)

(8)

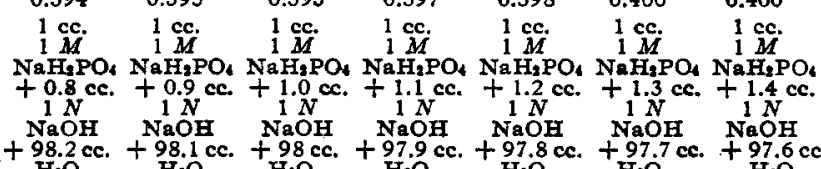

$+98.2 \mathrm{cc}+98.1 \mathrm{cc}+98 \mathrm{cc}+97.9 \mathrm{cc}+97.8 \mathrm{cc}+97.7 \mathrm{cc}+97.6 \mathrm{cc}$

Hours in the solution.

20

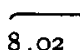

Gain in parts

$\mathrm{H}_{3} \mathrm{O}$

$\mathbf{H}$

44

8.02

8. 12

8.17

8.37

8.20

9.80

10.25

9.45

9.57

$9 \cdot 70$

9.84

9.60

11.03

I I. 30

Solution No.. .

(9)

(I0)

(II)

(12)

(13)

(I4)

(I5) 


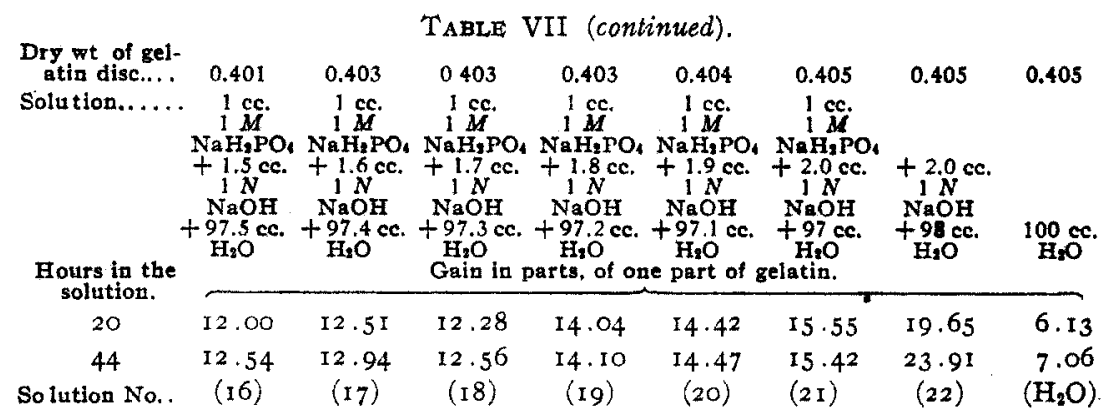

A set of experiments was next made by beginning with a fixed concentration of acid $(0.03 N)$ and adding to this progressively greater amounts of alkali. Under such circumstances there are seen, of course, the double effects of decrease in concentration of free acid with increase in amount of phosphate present. The general shape of the water absorption curve as shown in Fig. 8 and Table VIII is, however, that familiar to us from the previous experiments. A low point is again found in the middle of the curve when one-third of the replaceable hydrogen of the phosphoric acid has been neutralized by alkali, in other words, when we deal with a solution which is practically pure monosodium phosphate. Addition of more alkali leads to increased swelling up to the high point at the right of the curve which represents the theoretically pure solution of trisodium phosphate.

TABLE VIII.

Gelatin-Phosphoric Acid with Increasing Amounts of Sodium Hydroxide.

\begin{tabular}{|c|c|c|c|c|c|c|c|c|}
\hline $\begin{array}{l}\text { Dry wt. of gel- } \\
\text { atin disc.... }\end{array}$ & 0.343 & 0.343 & 0.345 & 0.347 & 0.360 & 0.363 & 0.365 & 0.367 \\
\hline Solution..... & $\begin{array}{c}3 \mathrm{cc} . \\
1 N \\
\mathrm{H}_{8} \mathrm{PO} \\
+97 \mathrm{cc} . \\
\mathrm{H}_{2} \mathrm{O}\end{array}$ & $\begin{array}{c}3 \mathrm{cc} . \\
1 N \\
\mathrm{H}_{2} \mathrm{PO} \\
+0.1 \mathrm{cc} . \\
1 \mathrm{~N} \\
\mathrm{NaOH} \\
+96.9 \mathrm{cc} \\
\mathrm{H}_{8} \mathrm{O}\end{array}$ & $\begin{array}{c}3 \mathrm{cc} . \\
1 N \\
\mathrm{H}_{2} \mathrm{PO} \\
+0.2 \mathrm{cc} \\
1 \mathrm{~N} \\
\mathrm{NaOH} \\
+96.8 \mathrm{cc} \\
\mathrm{H} \mathrm{H}_{2} \mathrm{O} \\
\text { Gain in pe }\end{array}$ & $\begin{array}{c}3 \mathrm{cc} \\
1 \mathrm{~N} \\
\mathrm{H}_{1} \mathrm{PO}_{4} \\
+0.3 \mathrm{cc} . \\
1 \mathrm{~N} \\
\mathrm{NaOH} \\
+96.7 \mathrm{cc} \\
\mathrm{H}_{2} \mathrm{O} \\
\text { arts, of one }\end{array}$ & $\begin{array}{c}3 \mathrm{cc} . \\
1 \mathrm{~N} \\
\mathrm{H}_{2} \mathrm{PO} \\
+0.4 \mathrm{cc} \\
1 \mathrm{~N} \\
\mathrm{NaOH} \\
+96.6 \mathrm{cc} . \\
\mathrm{H} \mathrm{O} \\
\text { part of gel }\end{array}$ & $\begin{array}{c}3 \mathrm{cc} \\
1 N \\
\mathrm{H}_{3} \mathrm{PO}_{4} \\
+0.5 \mathrm{cc} \\
1 N \\
\mathrm{NaOH} \\
+96.5 \mathrm{cc} . \\
\mathrm{H}_{2} \mathrm{O} \\
\text { latin. }\end{array}$ & $\begin{array}{c}3 \mathrm{cc} \\
1 N \\
\mathrm{H}_{2} \mathrm{PO} \\
+0.6 \mathrm{cc} \\
11 N \\
\mathrm{NaOH} \\
+96.4 \mathrm{cc} \\
\mathrm{H}_{2} \mathrm{O}\end{array}$ & $\begin{array}{c}3 \mathrm{cc} . \\
1 N \\
\mathrm{H}_{3} \mathrm{PO} \\
+0.7 \mathrm{cc} . \\
1 \mathrm{~N} \\
\mathrm{NaOH} \\
+96.3 \mathrm{cc} . \\
\mathrm{H}_{3} \mathrm{O}\end{array}$ \\
\hline 20 & $69 \cdot 32$ & 62.85 & 58.65 & 58.45 & 46.60 & 43.00 & 31.00 & $22 \cdot 3 I$ \\
\hline $\begin{array}{c}44 \\
\text { olution No... }\end{array}$ & $\begin{array}{c}84.68 \\
\text { (I) }\end{array}$ & $\begin{array}{l}75 \cdot \mathrm{II} \\
(2)\end{array}$ & $\begin{array}{c}70.60 \\
(3)\end{array}$ & $\begin{array}{l}69.90 \\
(4)\end{array}$ & $\begin{array}{l}58.17 \\
(5)\end{array}$ & $\begin{array}{l}54 \cdot 16 \\
(6)\end{array}$ & $\begin{array}{l}42.20 \\
(7)\end{array}$ & $\begin{array}{l}33.87 \\
(8)\end{array}$ \\
\hline $\begin{array}{l}\text { Dry } \\
\text { at }\end{array}$ & 0.367 & 0.367 & 0.367 & 0.370 & 0.370 & 0.370 & 0.371 & 0.374 \\
\hline Solution. & $\begin{array}{r}3 \mathrm{cc} . \\
1 N \\
\mathrm{H}_{1} \mathrm{PO} \\
+0.8 \mathrm{cc} \\
1 \mathrm{~N} \\
\mathrm{NaOH} \\
+96.2 \mathrm{cc} . \\
\mathrm{H}_{2} \mathrm{O}\end{array}$ & $\begin{array}{r}3 \mathrm{cc} \\
1 \mathrm{~N} \\
\mathrm{H} \mathrm{PO} \\
+0.9 \mathrm{cc} . \\
1 \mathrm{~N} \\
\mathrm{NaOH} \\
+96.1 \mathrm{cc} \\
\mathrm{H}_{2} \mathrm{O}\end{array}$ & $\begin{array}{c}3 \mathrm{cc} \\
1 \mathrm{~N} \\
\mathrm{H}, \mathrm{PO} \\
+1.0 \mathrm{cc} . \\
1 \mathrm{~N} \\
\mathrm{NaOH} \\
+96.0 \mathrm{cc} . \\
\mathrm{H}, \mathrm{O} \\
\text { Gain in }\end{array}$ & $\begin{array}{c}3 \mathrm{cc} . \\
1 N \\
\mathrm{H} . \mathrm{PO} \\
+1.1 \mathrm{cc} \\
1 \mathrm{~N} \\
\mathrm{NaOH} \\
+95.9 \mathrm{cc} . \\
\mathrm{H}_{2} \mathrm{O}\end{array}$ & $\begin{array}{r}3 \mathrm{cc} \\
1 \mathrm{~N} \\
\mathrm{H} . \mathrm{PO} \\
+1.2 \mathrm{cc} \\
1 \mathrm{~N} \\
\mathrm{NaOH} \\
+95.8 \mathrm{cc} \\
\mathrm{H} \mathrm{O} \\
\text { part of } \mathrm{g}\end{array}$ & $\begin{array}{c}3 \mathrm{cc} . \\
1 N \\
\mathrm{H}_{2} \mathrm{PO} \\
+1.3 \mathrm{cc} \\
11 \mathrm{~N} \\
\mathrm{NaOH} \\
+95.7 \mathrm{cc} . \\
\mathrm{H}_{1} \mathrm{O} \\
\text { gelatin. }\end{array}$ & $\begin{array}{r}3 \mathrm{cc} . \\
1 N \\
\mathrm{HsPO} \\
+1.4 \mathrm{cc} . \\
1 \mathrm{~N} \\
\mathrm{NaOH} \\
+95.6 \mathrm{cc} . \\
\mathrm{H} . \mathrm{O}\end{array}$ & $\begin{array}{r}3 \mathrm{cc} . \\
1 \mathrm{~N} \\
\mathrm{H}, \mathrm{PO}^{2} . \\
+1.5 \mathrm{cc} . \\
1 \mathrm{~N} \\
\mathrm{NaOH} \\
+95.5 \mathrm{cc} . \\
\mathrm{HgO}\end{array}$ \\
\hline 20 & I5 30 & $8.3 I$ & 7.10 & 8.03 & 8.68 & 8.74 & $9 \cdot 35$ & 9.60 \\
\hline $\begin{array}{c}44 \\
\text { Solution No... }\end{array}$ & $\begin{array}{c}24 \cdot 32 \\
(9)\end{array}$ & $\begin{array}{c}11.28 \\
(10)\end{array}$ & $\begin{array}{l}8.27 \\
(I I)\end{array}$ & $\begin{array}{l}9.4 I \\
(\mathrm{I} 2)\end{array}$ & $\begin{array}{l}10.27 \\
\text { (13) }\end{array}$ & $\begin{array}{c}\text { I0. } 44 \\
\text { (14) }\end{array}$ & $\begin{array}{c}\text { II.I6 } \\
(15)\end{array}$ & $\begin{array}{c}11.56 \\
( \pm 6)\end{array}$ \\
\hline
\end{tabular}




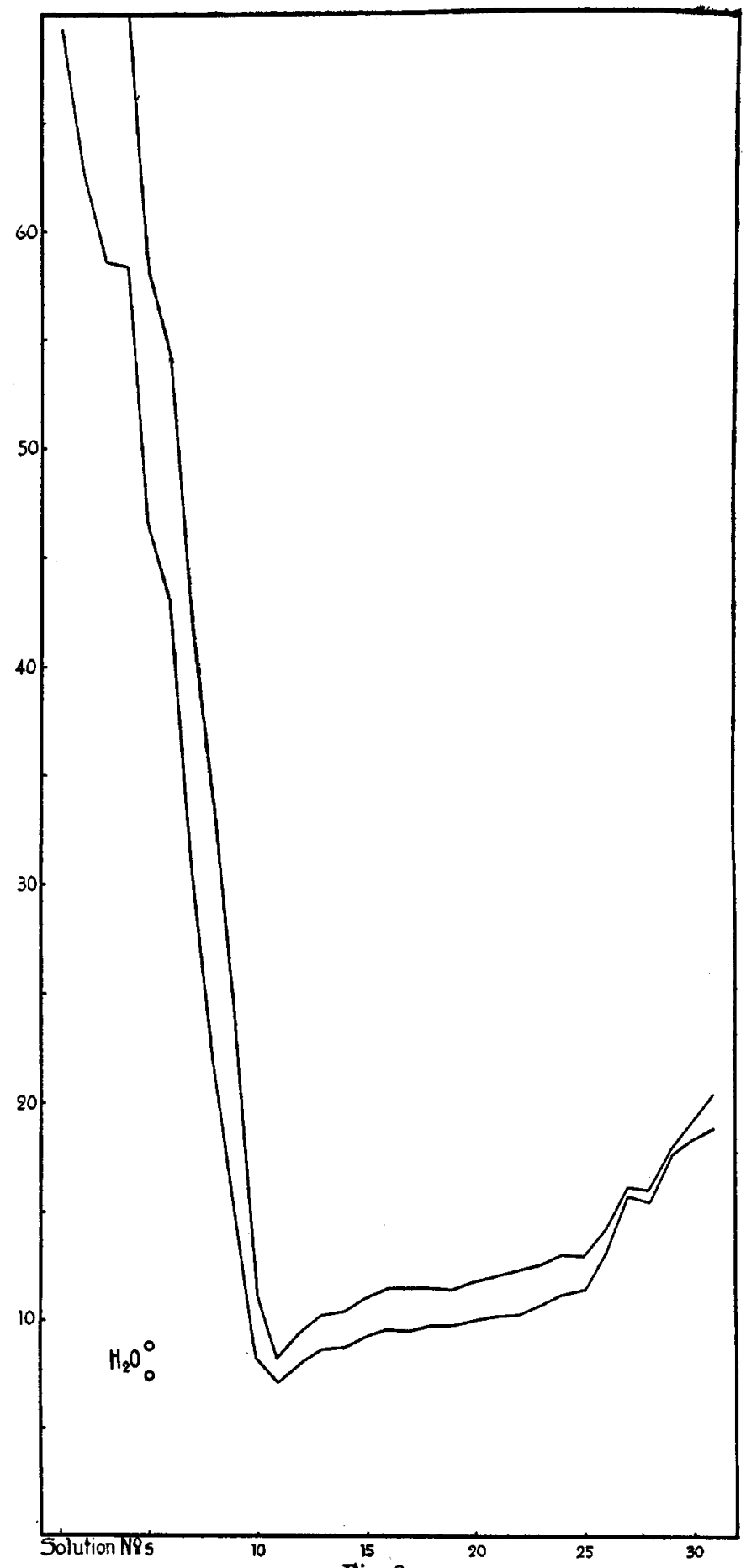

Fig. 8. 


\begin{tabular}{|c|c|c|c|c|c|c|c|c|}
\hline \multirow{2}{*}{$\begin{array}{c}\text { Dry of of gel- } \\
\text { atin disc.... } \\
\text { Solution..... }\end{array}$} & \multicolumn{3}{|c|}{ E VIIL } & & & & & \\
\hline & $\begin{array}{c}3.3 \mathrm{cc} \\
1 \mathrm{~N} \\
1 \mathrm{H} \\
+1.6 \mathrm{cc} \\
1 \mathrm{NO} \\
1 \mathrm{NaOH} \\
+95.4 \mathrm{cc} . \\
\mathrm{H}_{2} \mathrm{O}\end{array}$ & $\begin{array}{c}3 \mathrm{cc} \\
1 \mathrm{~N} \\
\mathrm{H}_{3} \mathrm{PO}_{4} \\
+1.7 \mathrm{cc} . \\
11 \mathrm{~N} \\
\mathrm{NaOH} \\
+95.3 \mathrm{cc} . \\
\mathrm{H}_{2} \mathrm{O}\end{array}$ & $\begin{array}{r}3.383 \\
3 \mathrm{cc} . \\
\mathrm{N} \\
\mathrm{H}_{\mathrm{s}} \mathrm{PO}_{4} \\
+1.8 \mathrm{cc} . \\
1 \mathrm{~N} \\
\mathrm{NaOH} \\
+95.2 \mathrm{cc} . \\
\mathrm{H}_{2} \mathrm{O} \\
\text { Gain }\end{array}$ & $\begin{array}{r}3 \mathrm{cc} \\
1 \mathrm{~N} \\
\mathrm{H}_{8} \mathrm{PO}_{4} \\
+1.9 \mathrm{cc} . \\
1 \mathrm{~N} \\
\mathrm{NaOH} \\
+95.1 \mathrm{cc} \\
\mathrm{H}, \mathrm{O} \\
\text { in parts, of }\end{array}$ & $\begin{array}{r}3 \mathrm{cc} \\
1 \mathrm{~N} \\
\mathrm{H}_{3} \mathrm{PO} \\
+2.0 \mathrm{cc} \\
1 \mathrm{~N} \\
\mathrm{NaOH} \\
+95.0 \mathrm{cc} \\
\mathrm{H}_{3} \mathrm{O} \\
\text { one part }\end{array}$ & $\begin{array}{c}3 \mathrm{cc} \\
1 \mathrm{~N} \\
\mathrm{H}_{8} \mathrm{PO}_{4} \\
+2.1 \mathrm{cc} . \\
1 \mathrm{~N} \\
\mathrm{NaOH} \\
+94.9 \mathrm{cc} \\
\mathrm{H} . \mathrm{C} \\
\text { of gelatin. }\end{array}$ & $\begin{array}{c}3.388 \\
3 \mathrm{cc} . \\
1 \mathrm{~N} \\
\mathrm{H}_{8} \mathrm{PO} \\
+2.2 \mathrm{cc} . \\
1 \mathrm{~N} \\
\mathrm{NaOH} \\
+94.8 \mathrm{cc} . \\
\mathrm{H}_{2} \mathrm{O}\end{array}$ & $\begin{array}{c}3.388 \\
3 \mathrm{cc} \\
1 \mathrm{~N} \\
\mathrm{H}_{8} \mathrm{PO}_{4} \\
+2.3 \mathrm{cc} . \\
1 \mathrm{~N} \\
\mathrm{NaOH} \\
+94.7 \mathrm{cc} . \\
\mathrm{H}_{3} \mathrm{O}\end{array}$ \\
\hline 20 & 9.49 & $9.8 \mathrm{r}$ & $9.8 \mathrm{I}$ & 10.00 & 10.23 & 10. 26 & 10.80 & II .28 \\
\hline $\begin{array}{c}44 \\
\text { dution No... }\end{array}$ & $\begin{array}{l}\text { II. } 3 \mathrm{I} \\
(\mathrm{I} 7)\end{array}$ & $\begin{array}{l}\text { II. } 60 \\
\text { (1 } 8 \text { ) }\end{array}$ & $\begin{array}{l}\text { I I.57 } \\
\text { (19) }\end{array}$ & $\begin{array}{l}\text { I1.90 } \\
(20)\end{array}$ & $\begin{array}{l}\mathrm{r}_{2} \cdot \mathrm{r}_{4} \\
(2 \mathrm{I})\end{array}$ & $\begin{array}{l}12.40 \\
(22)\end{array}$ & $\begin{array}{c}12.6 \mathrm{r} \\
(23)\end{array}$ & $\begin{array}{c}\text { I3. I } 2 \\
(24)\end{array}$ \\
\hline $\begin{array}{l}\text { ry wt. in } \\
\text { atin.... } \\
\text { slution.. }\end{array}$ & $\begin{array}{c}0.388 \\
3 \mathrm{cc} \\
1 \mathrm{~N} \\
\mathrm{H}_{3} \mathrm{PO}_{4} \\
+2.4 \mathrm{cc} \\
1 \mathrm{~N} \\
\mathrm{NaOH} \\
+94.6 \mathrm{cc} . \\
\mathrm{H}_{4} \mathrm{O}\end{array}$ & $\begin{array}{c}0.388 \\
3 \mathrm{cc} \\
1 \mathrm{~N} \\
\mathrm{H}_{3} \mathrm{PO} \\
+2.5 \mathrm{cc} . \\
1 \mathrm{~N} \\
\mathrm{NaOH} \\
+94.5 \mathrm{cc} . \\
\mathrm{H}_{8} \mathrm{O}\end{array}$ & $\begin{array}{c}0.389 \\
3 \mathrm{cc} \\
1 \mathrm{~N} \\
\mathrm{H}_{3} \mathrm{PO}_{4} \\
+2.6 \mathrm{cc} . \\
1 \mathrm{~N} \\
\mathrm{NaOH}^{2} \\
+94.4 \mathrm{cc} . \\
\mathrm{H}_{2} \mathrm{O} \\
\text { Gain in }\end{array}$ & $\begin{array}{c}0.391 \\
3 \mathrm{cc} \\
1 N \\
\mathrm{H}_{2} \mathrm{PO}_{4} \\
+2.7 \mathrm{cc} . \\
1 \mathrm{~N} \\
\mathrm{NaOH}^{2} \\
+94.3 \mathrm{cc} \\
\mathrm{H}_{2} \mathrm{O} \\
\text { parts, of on }\end{array}$ & $\begin{array}{r}0.391 \\
3 \mathrm{cc} . \\
1 \mathrm{~N} \\
\mathrm{FH}_{2} \mathrm{PO} \\
+2.8 \mathrm{cc} . \\
1 \mathrm{~N} \\
\mathrm{NaOH} \\
+94.2 \mathrm{cc} . \\
\mathrm{H}_{2} \mathrm{O} \\
\text { e part of }\end{array}$ & $\begin{array}{c}0.392 \\
3 \mathrm{cc} . \\
1 \mathrm{~N} \\
\mathrm{H}_{3} \mathrm{PO} \\
+2.9 \mathrm{cc} . \\
1 \mathrm{~N} \\
\mathrm{NaOH} \\
+94.1 \mathrm{cc} . \\
\mathrm{H}_{2} \mathrm{O} \\
\text { gelatin. }\end{array}$ & $\begin{array}{c}0.394 \\
3 \mathrm{cc} \\
1 \stackrel{N}{1} \\
\mathrm{H}_{3} \mathrm{PO}_{4} \\
+3.0 \mathrm{cc} . \\
1 . N \\
\mathrm{NaOH} \\
+94 \mathrm{cc} \\
\mathrm{H}_{2} \mathrm{O}\end{array}$ & $\begin{array}{c}100 \mathrm{cc} . \\
\mathrm{H}_{2} \mathrm{O}\end{array}$ \\
\hline 20 & II.5 & 13.14 & 1 & & & 40 & 19 & $7 \cdot 45$ \\
\hline $\begin{array}{c}44 \\
\text { llution No. }\end{array}$ & $\begin{array}{c}13.00 \\
(25)\end{array}$ & $\begin{array}{c}14.24 \\
(26)\end{array}$ & $\begin{array}{c}16.25 \\
(27)\end{array}$ & $\begin{array}{l}16.17 \\
(28)\end{array}$ & $\begin{array}{c}18.03 \\
(29)\end{array}$ & $\begin{array}{l}19.37 \\
(30)\end{array}$ & $\begin{array}{c}20.50 \\
(31)\end{array}$ & $\begin{array}{r}8.80 \\
\left(\mathrm{H}_{2} \mathrm{O}\right)\end{array}$ \\
\hline
\end{tabular}

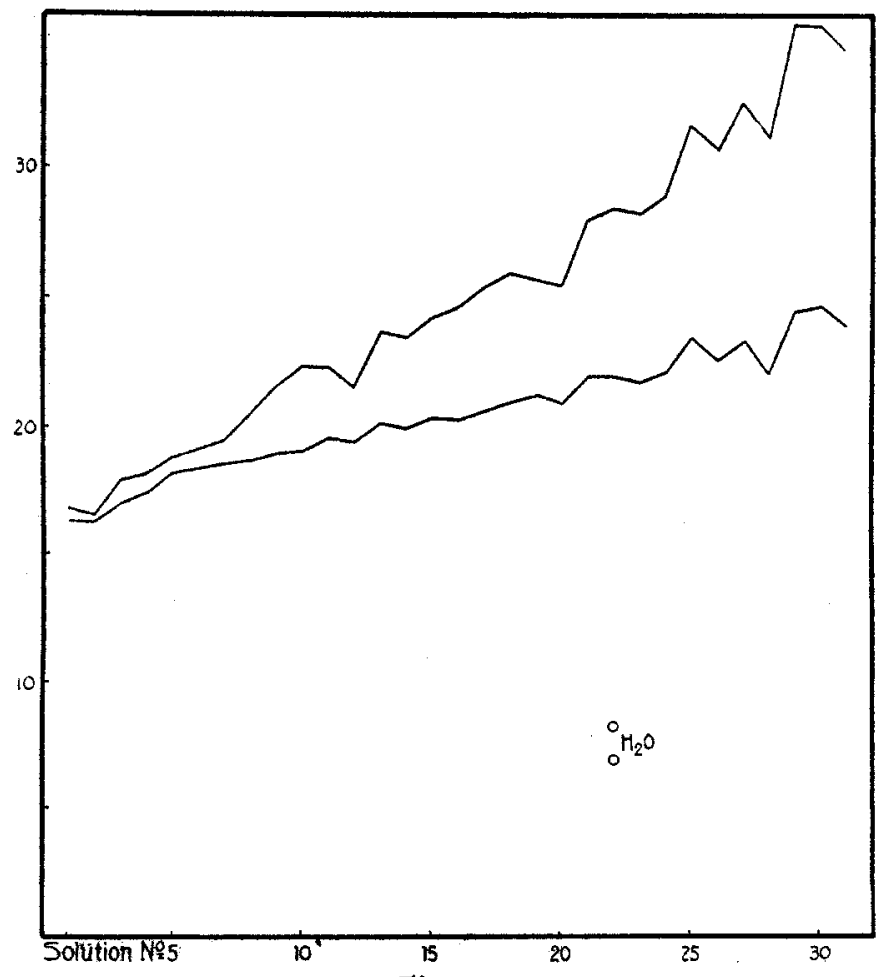

Fig. 9. 
A final series of phosphate experiments is shown in Fig. 9 and Table IX. Here a fixed amount of alkali $(0.03 N)$ was neutralized by adding gradually increasing amounts of phosphoric acid. There are shown, in other words, the effects of simultaneous reduction of acid with increase in amount of phosphate present. The water absorption curve falls as the neutralization progresses, until a low point is attained in the theoretically pure solution of trisodium phosphate.

TABLE IX.

Gelatin-Sodium Hydroxide with Decreasing Amounts of Phosphoric Acid.

Dry wt of gel-

\begin{tabular}{|c|c|c|c|c|c|c|c|c|}
\hline atio discs.. & $\begin{array}{c}0.347 \\
3 \mathrm{cc} .1 \mathrm{~N} \\
\mathrm{NaOH} \\
+3.0 \mathrm{cc} \\
1 \mathrm{~N} \\
\mathrm{HgPO}_{4} \\
+94 \mathrm{cc} . \\
\mathrm{H}_{2} \mathrm{O}\end{array}$ & $\begin{array}{c}0.346 \\
3 \mathrm{cc} .1 \mathrm{~N} \\
\mathrm{NaOH} \\
+2.9 \mathrm{cc} . \\
1 \mathrm{~N} \\
\mathrm{H}_{8} \mathrm{PO} \\
+94.1 \mathrm{cc} . \\
\mathrm{H} \mathrm{H}_{2} \mathrm{O}\end{array}$ & $\begin{array}{c}0.343 \\
3 \mathrm{cc} .1 \mathrm{~N} \\
\mathrm{NaOH} \\
+2.8 \mathrm{cc} . \\
1 \mathrm{~N} \\
\mathrm{H}_{9} \mathrm{PO}_{4} \\
+94.2 \mathrm{cc} . \\
\mathrm{H}_{2} \mathrm{O} \\
\mathrm{Gain} \text { in }\end{array}$ & $\begin{array}{c}0.342 \\
3 \mathrm{cc} .1 \mathrm{~N} \\
\mathrm{NaOH} \\
+2.7 \mathrm{cc} . \\
1 \mathrm{~N} \\
\mathrm{H}_{8} \mathrm{PO}_{4} \\
+94.3 \mathrm{cc} . \\
\mathrm{H}_{2} \mathrm{O} \\
\text { parts, of on }\end{array}$ & $\begin{array}{c}0.341 \\
3 \mathrm{cc}, 1 \mathrm{~N} \\
\mathrm{NaOH} \\
+2.6 \mathrm{cc} . \\
1 \mathrm{~N} \\
\mathrm{H}_{8} \mathrm{PO} \\
+94.4 \mathrm{cc} . \\
\mathrm{H} \text {. } \\
\text { ne part of }\end{array}$ & $\begin{array}{c}0.341 \\
3 \mathrm{cc} .1 \mathrm{~N} \\
\mathrm{NaOH} \\
+2.5 \mathrm{cc} \\
1 \mathrm{~N} \\
\mathrm{H}_{2} \mathrm{PO} \\
+94.5 \mathrm{cc} . \\
\mathrm{H}_{2} \mathrm{O} \\
\text { gelatin. }\end{array}$ & $\begin{array}{c}0.340 \\
3 \mathrm{cc} .1 \mathrm{~N} \\
\mathrm{NaOH} \\
+2.4 \mathrm{cc} \\
1 \mathrm{~N} \\
\mathrm{HzPO} \\
+94.6 \mathrm{cc} . \\
\mathrm{H}_{2} \mathrm{O}\end{array}$ & $\begin{array}{c}0.340 \\
3 \mathrm{cc} 1 \mathrm{~N} \\
\mathrm{NaOH} \\
+2.3 \mathrm{cc} . \\
1 \mathrm{~N} \\
\mathrm{H}_{3} \mathrm{PO} \\
+94.7 \mathrm{cc} . \\
\mathrm{H}_{2} \mathrm{O}\end{array}$ \\
\hline 20 & I 6.46 & I 6. & I 7.17 & 17.55 & I 8.32 & I 8. & & I8.85 \\
\hline$\stackrel{44}{\text { olution No... }}$ & $\begin{array}{c}\text { I } 7.00 \\
(\mathrm{I})\end{array}$ & $\begin{array}{c}16.69 \\
(2)\end{array}$ & $\begin{array}{l}\text { I } 8.02 \\
(3)\end{array}$ & $\begin{array}{c}18.30 \\
(4)\end{array}$ & $\begin{array}{c}\mathrm{I} 8.82 \\
(5)\end{array}$ & $\begin{array}{l}18.80 \\
(6)\end{array}$ & (7) & $\begin{array}{c}20.53 \\
(8)\end{array}$ \\
\hline ry & . $\quad 0.340$ & & 0.338 & 0.338 & 0.338 & & & \\
\hline $\begin{array}{l}\text { Hours ir } \\
\text { soluti }\end{array}$ & $\begin{array}{c}3 \mathrm{cc} 1 \mathrm{~N} \\
\mathrm{NaOH} \\
+2.2 \mathrm{cc} . \\
1 \mathrm{~N} \\
\mathrm{H}_{3} \mathrm{PO}_{4} \\
+94.8 \mathrm{cc} . \\
\mathrm{H}_{2} \mathrm{O}\end{array}$ & $\begin{array}{c}3 \mathrm{cc} 1 \mathrm{~N} \\
\mathrm{NaOH} \\
+2.1 \mathrm{cc} \\
1 \mathrm{~N} \\
\mathrm{H}_{3} \mathrm{PO}_{4} \\
+94.9 \mathrm{cc} . \\
\mathrm{H}_{2} \mathrm{O}\end{array}$ & $\begin{array}{l}3 \mathrm{cc} 1 \mathrm{~N} \\
\mathrm{NaOH} \\
+2.0 \mathrm{cc} \\
1 \mathrm{~N} \\
\mathrm{H}_{3} \mathrm{PO} \\
+95 \mathrm{cc} \\
\mathrm{H} \mathrm{H}_{2} \mathrm{O} \\
\mathrm{Gain} \text { in }\end{array}$ & $\begin{array}{c}3 \mathrm{cc} .1 \mathrm{~N} \\
\mathrm{NaOH} \\
+1.9 \mathrm{cc} . \\
1 \mathrm{~N} \\
\mathrm{H}_{2} \mathrm{PO}_{4} \\
+95.1 \mathrm{cc} . \\
\mathrm{H}_{2} \mathrm{O} \text {. } \\
\text { parts, of on }\end{array}$ & $\begin{array}{c}3 \mathrm{cc} 1 \mathrm{~N} \\
\mathrm{NaOH} \\
+1.8 \mathrm{cc} . \\
1 \mathrm{~N} \\
\mathrm{HzPO} \\
+95.2 \mathrm{cc} \\
\mathrm{HzO} \\
\text { ne part of }\end{array}$ & $\begin{array}{c}3 \mathrm{cc.} 1 \mathrm{~N} \\
\mathrm{NaOH} \\
+1.7 \mathrm{cc} \\
1 \mathrm{~N} \\
\mathrm{H}_{3} \mathrm{PO}_{4} \\
+95.3 \mathrm{cc} . \\
\mathrm{H}_{3} \mathrm{O} \\
\text { gelatin. }\end{array}$ & $\begin{array}{c}3 \mathrm{cc} .1 \mathrm{~N} \\
\mathrm{NaOH} \\
+1.6 \mathrm{cc} \\
1 . N \\
\mathrm{H}_{8} \mathrm{PO} \\
+95.4 \mathrm{cc} . \\
\mathrm{H}_{3} \mathrm{O}\end{array}$ & $\begin{array}{c}3 \mathrm{cc} 1 \mathrm{~N} \\
\mathrm{NaOH} \\
+1.5 \mathrm{cc} . \\
1 . N \\
\mathrm{H}_{2} \mathrm{PO} \\
+95.5 \mathrm{cc} . \\
\mathrm{H}_{9} \mathrm{O}\end{array}$ \\
\hline 20 & $\times 9$. & I9. & 19.65 & 19.47 & 20.25 & 20 & & $20 \cdot 35$ \\
\hline 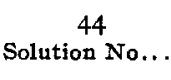 & $\begin{array}{c}21.60 \\
\quad(9)\end{array}$ & $\begin{array}{l}22.42 \\
\text { (10) }\end{array}$ & $\begin{array}{c}22.43 \\
(I I)\end{array}$ & $\begin{array}{l}21.65 \\
(12)\end{array}$ & $\begin{array}{c}23 \cdot 76 \\
(13)\end{array}$ & $\begin{array}{c}23 \cdot 55 \\
(14)\end{array}$ & $\begin{array}{c}24 \cdot 37 \\
(15)\end{array}$ & $\begin{array}{c}24 \cdot 70 \\
(16)\end{array}$ \\
\hline $\begin{array}{l}\text { ry } \\
\text { ati }\end{array}$ & 0.32 & & 0.335 & 0.335 & 0.334 & 0.332 & & \\
\hline lution & $\begin{array}{c}3 \mathrm{cc} 1 \mathrm{~N} \\
\mathrm{NaOH} \\
+1.4 \mathrm{cc} \\
1 \mathrm{~N} \\
\mathrm{H}_{8} \mathrm{PO}_{4} \\
+95.6 \mathrm{cc} \\
\mathrm{H}_{2} \mathrm{O}\end{array}$ & $\begin{array}{c}3 \mathrm{cc} 1 \mathrm{~N} \\
\mathrm{NaOH} \\
+1.3 \mathrm{cc} \\
1 \mathrm{cc} \\
\mathrm{H}_{3} \mathrm{PO} \\
+95.7 \mathrm{cc} . \\
\mathrm{H}_{3} \mathrm{O}\end{array}$ & $\begin{array}{c}3 \mathrm{cc} 1 \mathrm{~N} \\
\mathrm{NaOH} \\
+1.2 \mathrm{cc} \\
1 \mathrm{~N} \\
\mathrm{H}_{8} \mathrm{PO} \\
+95.8 \mathrm{cc} . \\
\mathrm{H}_{2} \mathrm{O} \\
\text { Gain in }\end{array}$ & $\begin{array}{c}3 \mathrm{cc} .1 \mathrm{~N} \\
\mathrm{NaOH} \\
+1.1 \mathrm{cc} \\
1 \mathrm{~N} \\
\mathrm{HzPO} \\
+95.9 \mathrm{cc} \\
\mathrm{H}, \mathrm{O} \\
\text { parts, of of }\end{array}$ & $\begin{array}{c}3 \mathrm{cc} .1 \mathrm{~N} \\
\mathrm{NaOH} \\
+1.0 \mathrm{cc} \\
1 \mathrm{~N} \\
\mathrm{H}_{2} \mathrm{PO}_{4} \\
+96 \mathrm{cc} \\
\mathrm{H}_{2} \mathrm{O}\end{array}$ & $\begin{array}{l}3 \mathrm{cc} .1 N \\
\mathrm{NaOH} \\
+0.9 \mathrm{cc} \\
1 \mathrm{~N} \\
\mathrm{H} . \mathrm{PO} \\
+96.1 \mathrm{cc} \\
\mathrm{H}_{3} \mathrm{O}\end{array}$ & $\begin{array}{c}3 \mathrm{cc} .1 \mathrm{~N} \\
\mathrm{NaOH} \\
+0.8 \mathrm{cc} \\
1 \mathrm{~N} \\
\mathrm{H} . \mathrm{PO} \\
+96.2 \mathrm{cc} . \\
\mathrm{H}_{2} \mathrm{O}\end{array}$ & $\begin{array}{c}3 \mathrm{cc} .1 \mathrm{~N} \\
\mathrm{NaOH} \\
+0.7 \mathrm{cc} . \\
1 \mathrm{~N} \\
\mathrm{H}_{2} \mathrm{PO} \\
+96.3 \mathrm{cc} . \\
\mathrm{H}_{3} \mathrm{O}\end{array}$ \\
\hline 20 & 20.77 & & 2 & .97 & 22.06 & 22 & & .21 \\
\hline$\stackrel{44}{\text { lution No... }}$ & $\begin{array}{c}25 \cdot 55 \\
(17)\end{array}$ & $\begin{array}{c}26.00 \\
\text { (I } 8)\end{array}$ & $\begin{array}{c}25.85 \\
(19)\end{array}$ & $\begin{array}{c}25 \cdot 58 \\
(20)\end{array}$ & $\begin{array}{c}28.14 \\
(2 \mathrm{I})\end{array}$ & $\begin{array}{c}28.50 \\
(22)\end{array}$ & (23) & $\begin{array}{c}29.03 \\
(24)\end{array}$ \\
\hline atin discs... & 0.330 & & & 0.023 & 0.329 & 0.020 & 0.327 & 0.347 \\
\hline 1) & $\begin{array}{c}3 \mathrm{cc} .1 \mathrm{~N} \\
\mathrm{NaOH} \\
+0.6 \mathrm{cc} \\
1 \mathrm{~N} \\
\mathrm{H}_{2} \mathrm{PO} \\
+96.4 \mathrm{cc} \\
\mathrm{H}_{2} \mathrm{O}\end{array}$ & $\begin{array}{c}3 \mathrm{cc} 1 \mathrm{~N} \\
\mathrm{NaOH} \\
+0.5 \mathrm{cc} . \\
1 \mathrm{~N} \\
\mathrm{H}_{3} \mathrm{PO} \\
+96.5 \mathrm{cc} . \\
\mathrm{H}_{2} \mathrm{O}\end{array}$ & $\begin{array}{l}3 \mathrm{cc} 1 \mathrm{~N} \\
\mathrm{NaOH} \\
+0.4 \mathrm{cc} \\
1 \mathrm{~N} \\
\mathrm{H}_{3} \mathrm{PO} \\
+96.6 \mathrm{cc} . \\
\mathrm{H}_{2} \mathrm{O} \\
\text { Gain in }\end{array}$ & $\begin{array}{c}3 \mathrm{cc} 1 \mathrm{~N} \\
\mathrm{NaOH} \\
+0.3 \mathrm{cc} \\
1 \mathrm{~N} \\
\mathrm{H} \mathrm{PO} \\
+96.7 \mathrm{cc} \\
\mathrm{H}_{8} \mathrm{O}\end{array}$ & $\begin{array}{c}3 \mathrm{cc} 1 \mathrm{~N} \\
\mathrm{NaOH} \\
+0.2 \mathrm{cc} \\
1 \mathrm{~N} \\
\mathrm{H}_{8} \mathrm{PO} \\
+96.8 \mathrm{cc} . \\
\mathrm{H} . \mathrm{O}\end{array}$ & $\begin{array}{c}3 \mathrm{cc} 1 \mathrm{~N} \\
\mathrm{NaOH} \\
+0.1 \mathrm{cc} . \\
1 . \mathrm{N} \\
\mathrm{H} \mathrm{HO}_{4} \\
+96.9 \mathrm{cc} . \\
\mathrm{H} \mathrm{H}_{2} \mathrm{O}\end{array}$ & $\begin{array}{c}3 \mathrm{cc} . \\
1 \mathrm{~N} \\
\mathrm{NaOH} \\
+97 \mathrm{cc} . \\
\mathrm{H}_{4} \mathrm{O}\end{array}$ & $\begin{array}{l}100 \mathrm{cc} . \\
\mathrm{H}_{\$} \mathrm{O}\end{array}$ \\
\hline 20 & $23 \cdot 56$ & & $23 \cdot 4$ & 22.13 & 22 & $24 \cdot 73$ & 24.00 & 0.94 \\
\hline 44 & $\begin{array}{c}31.80 \\
(25)\end{array}$ & $\begin{array}{c}30.8 \mathrm{I} \\
(26)\end{array}$ & $\begin{array}{c}32.66 \\
(27)\end{array}$ & $\begin{array}{c}31.26 \\
(28)\end{array}$ & $\begin{array}{c}35.68 \\
(29)\end{array}$ & $\begin{array}{c}35.6 \mathrm{I} \\
(30)\end{array}$ & $\begin{array}{c}34 \cdot 64 \\
(31)\end{array}$ & $\begin{array}{r}8.33 \\
\left(\mathrm{H}_{2} \mathrm{O}\right)\end{array}$ \\
\hline
\end{tabular}




\section{Experiments with Citrate Mixtures.}

We studied next the effects of citric acid and the citrates upon the swelling of gelatin. The citrate solutions were prepared by adding to each other the theoretically necessary amounts of carefully standardized citric acid and sodium hydroxide solutions.

In Figs. 10, II and 12, which portray graphically the experimental findings contained in Tables X, XI and XII, are shown the effects, respectively, of mono-, di-, and trisodium citrate in different concentrations
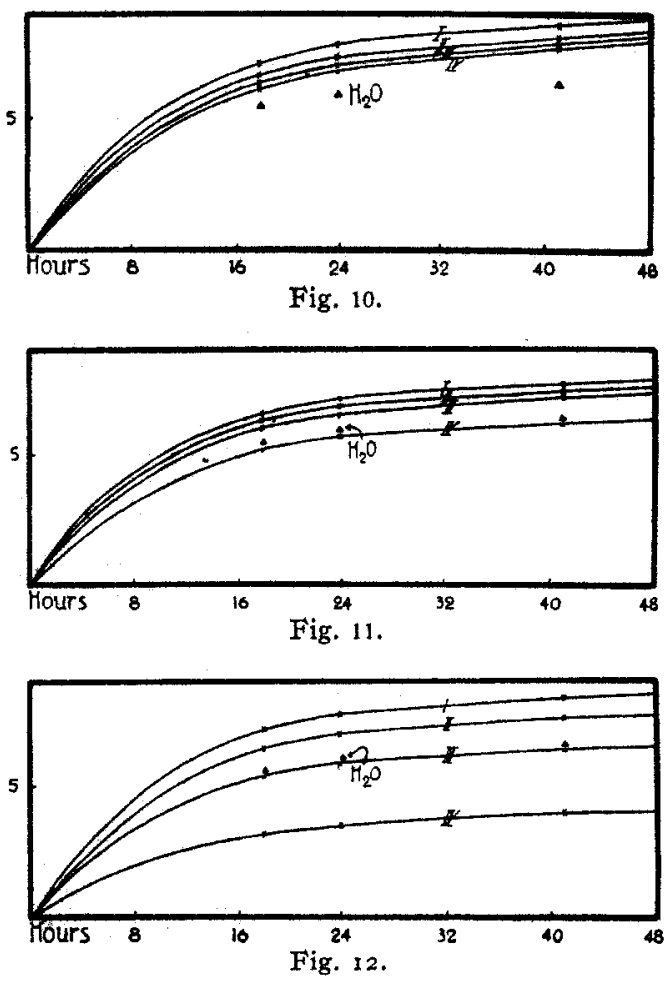
upon the swelling of gelatin. As evident in Fig. 1o, monosodium citrate in all the concentrations used increases the amount of water absorbed by gelatin over and above the amount absorbed in pure water. The same is true of the lower concentrations of equimolar solutions of disodium citrate. For both of these salts there is a progressive decrease in swelling with increase in the concentration of the salt. As evident in the lowermost curve of Fig. I I, a sufficiently high concentration of disodium citrate makes gelatin swell even less than in pure water. The effects of trisodium citrate are shown in Fig. 12. Low concentrations of this salt also increase the amount of water absorption over the amount absorbed in pure water, but with increasing concentration there is less and less swelling, until the amounts of water absorbed in the higher concentrations of the trisodium citrate are distinctly less than in pure water.

Having determined in this fashion the effects of different concentrations of the different citrates, we investigated the swelling of gelatin in citrate mixtures varying from the extreme on the one side of pure citric acid through equimolar concentrations of mono-, di- and trisodium citrate to pure sodium hydroxide upon the other. The results as shown in Fig. 13 and Table XIII are self-explanatory. The three curves show the amounts of water absorbed at the end of 24,48 and 72 hours. As 
TABLE X.

Gelatin-Monosodium Citrate.

\begin{tabular}{|c|c|c|c|c|c|}
\hline $\begin{array}{l}\text { Dry wt. of gelatin discs..... } \\
\text { Solution. } \ldots \ldots \ldots \ldots \ldots \ldots \ldots \ldots\end{array}$ & $\begin{array}{c}0.370 \\
5 \text { cc. } 1 \mathrm{M} \\
\text { monosod. } \\
\text { citrate } \\
+95 \mathrm{cc} . \\
\mathrm{H} . \mathrm{O}\end{array}$ & $\begin{array}{c}0.373 \\
15 \text { cc. } 1 M \\
\text { monosod. } \\
\text { citrate } \\
+85 \mathrm{cc} . \\
\text { H, } \\
\text { Gain in parts }\end{array}$ & $\begin{array}{c}0.373 \\
25 \text { cc. } 1 \mathrm{M} \\
\text { monosod. } \\
\text { cittate } \\
+75 \mathrm{cc} . \\
\mathrm{HzO} \\
\text { of one pax }\end{array}$ & $\begin{array}{c}0.373 \\
50 \text { cc. } 1 \mathrm{M} \\
\text { monosod. } \\
\text { citrate } \\
+50 \mathrm{cc} . \\
\mathrm{HzO} \\
\text { t of gelatin. }\end{array}$ & $\begin{array}{l}100 \mathrm{cc} . \\
\mathrm{H}, \mathrm{O}\end{array}$ \\
\hline 18 & 7.17 & 6.74 & 6.20 & 6.23 & $5 \cdot 52$ \\
\hline $\begin{array}{l}24 \\
4 I\end{array}$ & $\begin{array}{l}7.91 \\
8.69\end{array}$ & $\begin{array}{l}7.47 \\
8.16\end{array}$ & $\begin{array}{l}7.11 \\
8.27\end{array}$ & $\begin{array}{l}7.12 \\
8.01\end{array}$ & $\begin{array}{l}5 \cdot 94 \\
6.35\end{array}$ \\
\hline $\begin{array}{l}48 \\
66\end{array}$ & $\begin{array}{l}8.92 \\
9.30\end{array}$ & $\begin{array}{l}8.44 \\
8.81\end{array}$ & $\begin{array}{l}8.59 \\
9.08\end{array}$ & $\begin{array}{l}8.40 \\
9.00\end{array}$ & $\begin{array}{l}6.52 \\
6.84\end{array}$ \\
\hline 71 & 9.35 & 8.85 & 9.12 & 9. II & 6.90 \\
\hline $\begin{array}{c}91 \\
\text { Curve designation. }\end{array}$ & $\begin{array}{l}9.5 \mathrm{I} \\
(\mathrm{I})\end{array}$ & $\begin{array}{c}8.88 \\
\text { (II) }\end{array}$ & $\begin{array}{l}9.41 \\
\text { (III) }\end{array}$ & $\begin{array}{l}9.28 \\
\text { (IV) }\end{array}$ & $\begin{array}{l}7.27 \\
\left(\mathrm{H}_{2} \mathrm{O}\right)\end{array}$ \\
\hline
\end{tabular}

TABLE XI.

Gelatin-Disodium Citrate.

\begin{tabular}{|c|c|c|c|c|c|}
\hline $\begin{array}{l}\text { Dry wt, of gelatin discs. } \ldots \ldots \\
\text { Solution. } \ldots \ldots \ldots \ldots \ldots \ldots \ldots \cdots\end{array}$ & $\begin{array}{c}0.365 \\
5 \text { cc. } 1 M \\
\text { disod. } \\
\text { citrate } \\
+95 \mathrm{cc} . \\
\text { E. }\end{array}$ & $\begin{array}{c}0.367 \\
15 \text { cc. } 1 M \\
\text { disod. } \\
\text { citrate } \\
+85 \mathrm{cc} . \\
\mathrm{H}_{2} \mathrm{O} \\
\text { Gain in parts }\end{array}$ & $\begin{array}{c}0.369 \\
25 \text { cc. } 1 M \\
\text { disod. } \\
\text { citrate } \\
+75 \mathrm{cc} \text {. } \\
\text { H, } \\
\text { of one par }\end{array}$ & $\begin{array}{c}0.369 \\
50 \text { cc. } 1 \mathrm{M} \\
\text { disod. } \\
\text { citrate } \\
+50 \mathrm{cc} . \\
\text { H.O } \\
+ \text { of gelatin. }\end{array}$ & $\begin{array}{c}100 \text { cc. } \\
\mathrm{H}_{9} \mathrm{O}\end{array}$ \\
\hline 18 & 6.39 & 6.38 & $5 . I I$ & 5.12 & $5 \cdot 47$ \\
\hline 24 & 6.88 & 6.92 & 6.44 & $5 \cdot 7^{8}$ & 5.92 \\
\hline 41 & $7 \cdot 34$ & $7 \cdot 4 \mathrm{I}$ & 7.26 & 6.24 & 6.31 \\
\hline 48 & $7 \cdot 77$ & 7.58 & $7 \cdot 49$ & 6.48 & 6.49 \\
\hline 66 & $7 \cdot 70$ & 7.83 & $7 \cdot 74$ & 6.74 & 6.89 \\
\hline 71 & $7 \cdot 76$ & 7.84 & $7 \cdot 71$ & 6.77 & 6.96 \\
\hline $\begin{array}{l}9 I \\
\text { Curve designation }\end{array}$ & $\begin{array}{l}7.82 \\
\text { (I) }\end{array}$ & $\begin{array}{r}7.95 \\
\text { (II) }\end{array}$ & $\begin{array}{l}7.89 \\
\text { (III) }\end{array}$ & $\begin{array}{l}7.86 \\
\text { (IV) }\end{array}$ & $\begin{array}{l}7 \cdot 37 \\
\left(\mathrm{H}_{2} \mathrm{O}\right)\end{array}$ \\
\hline
\end{tabular}

TABLE XII.

Gelatin-Trisodium Citrate.

\begin{tabular}{|c|c|c|c|c|c|}
\hline $\begin{array}{l}\text { Dry wt. of gelatin discs...... } \\
\text { Solution. . . . . . } \ldots \ldots \ldots \ldots\end{array}$ & $\begin{array}{c}0.363 \\
5 \text { ce. } 1 \mathrm{M} \\
\text { trisod. } \\
\text { citrate } \\
+95 \mathrm{cc} . \\
\mathrm{H}_{2} \mathrm{O}\end{array}$ & $\begin{array}{c}0.363 \\
15 \text { cc. } 1 M \\
\text { trisod. } \\
\text { citrate } \\
+85 \mathrm{cc} . \\
\text { H: } \\
\text { Gain in parts }\end{array}$ & $\begin{array}{c}0.363 \\
25 \text { cc. } 1 M \\
\text { trisod. } \\
\text { citrate } \\
+75 \mathrm{cc} . \\
\mathrm{H}, \mathrm{O} \\
\text { of one par }\end{array}$ & $\begin{array}{c}0.364 \\
50 \text { ce. } 1 M \\
\text { trisod. } \\
\text { citrste } \\
+50 \text { cc. } \\
\text { HaO } \\
\text { tof gelatin. }\end{array}$ & $\begin{array}{c}100 \mathrm{cc} \\
\mathrm{H}_{3} \mathrm{O}\end{array}$ \\
\hline 18 & 7.12 & 6.42 & $5.5 \mathrm{I}$ & 3.12 & 5.53 \\
\hline 24 & $7 \cdot 75$ & 7.01 & 6.04 & $3 \cdot 52$ & $5 \cdot 98$ \\
\hline $4 I$ & 8.23 & $7.5 \mathrm{I}$ & $6.5 \mathrm{I}$ & 3.90 & 6.35 \\
\hline $4^{8}$ & 8.50 & 7.69 & 6.68 & 4.02 & $6.5^{8}$ \\
\hline 66 & 8.73 & 7.95 & 6.93 & $4.2 \mathrm{I}$ & 6.99 \\
\hline 71 & 8.73 & 7.96 & 6.96 & 4.23 & 7.08 \\
\hline $\begin{array}{c}91 \\
\text { Curve deaignation...... }\end{array}$ & $\begin{array}{l}8.8 \mathrm{I} \\
(\mathrm{I})\end{array}$ & $\begin{array}{r}8.06 \\
\text { (II) }\end{array}$ & $\begin{array}{l}7.04 \\
(\text { III })\end{array}$ & $\begin{array}{l}4 \cdot 30 \\
(I V)\end{array}$ & $\begin{array}{l}7.62 \\
\left(\mathrm{H}_{2} \mathrm{O}\right)\end{array}$ \\
\hline
\end{tabular}


readily evident, most swelling occurs in the pure citric acid, and less and less swelling as the pure citric acid gives way to the monosodium citrate.

TABLE XIII.

Gelatin-Citric Acid, to Citrates, to Sodium Hydroxide.

\begin{tabular}{|c|c|c|c|c|c|c|c|c|}
\hline $\begin{array}{c}\text { Dry wt, of gel- } \\
\text { atin discs... }\end{array}$ & 0.332 & 0.334 & 0.335 & 0.335 & 0.336 & 0.3 .38 & 0.339 & \\
\hline Solution...... & $\begin{array}{c}10 \mathrm{cc} \\
1 \mathrm{~N} \\
\text { citric } \\
\text { acid } \\
+90 \mathrm{ce} . \\
\mathrm{H}_{2} \mathrm{O}\end{array}$ & $\begin{array}{c}8 \mathrm{cc} . \\
1 \mathrm{~N} \\
\text { citric } \\
\text { acid } \\
+2 \mathrm{cc} \\
1 \mathrm{M} \\
\text { monosod. } \\
\text { citrate } \\
+90 \mathrm{cc} \\
\mathrm{H}_{2} \mathrm{O}\end{array}$ & $\begin{array}{c}6 \mathrm{cc} \\
1 \mathrm{~N} \\
\text { citric } \\
\text { acid } \\
+4 \mathrm{cc} . \\
1 \mathrm{M} \\
\text { monosod. } \\
\text { citrate } \\
+90 \mathrm{cc} \\
\mathrm{H} \mathrm{H}^{2} \\
\text { Gain in }\end{array}$ & $\begin{array}{l}4 \mathrm{cc} \\
1 \mathrm{~N} \\
\text { citric } \\
\text { acid } \\
+6 \mathrm{cc} . \\
1 \mathrm{M} \\
\text { monosod. } \\
\text { citrate } \\
+90 \mathrm{cc} \text {. } \\
\mathrm{H}_{2} \mathrm{O} \\
\text { parts, of }\end{array}$ & $\begin{array}{c}2 \mathrm{cc} . \\
1 N \\
\text { citric } \\
\text { acid } \\
+8 \mathrm{cc} . \\
1 M \\
\text { monosod. } \\
\text { citrate } \\
+90 \mathrm{cc} . \\
\mathrm{H}_{2} \mathrm{O} \\
\text { one part of }\end{array}$ & $\begin{array}{c}10 \mathrm{cc} \\
1 \mathrm{M} \\
\text { monosod. } \\
\text { citrate } \\
+90 \mathrm{cc} . \\
\mathrm{H}_{2} \mathrm{O} \\
\\
\text { gelatin. }\end{array}$ & $\begin{array}{c}8 \mathrm{cc} . \\
1 M \\
\text { monosod. } \\
\text { citrate } \\
+2 \mathrm{cc} . \\
1 \mathrm{M} \\
\text { disod. } \\
\text { citrate } \\
+90 \mathrm{cc} \\
\mathrm{H}_{2} \mathrm{O}\end{array}$ & \\
\hline 24 & 46.10 & 27.58 & 18.29 & I4. II & I I .08 & 9.02 & 7.66 & \\
\hline 48 & 51.08 & 33.14 & 22.71 & I 7.04 & I3.09 & 10.40 & 8.84 & \\
\hline 72 & 60.70 & 36.72 & 25.10 & 18.53 & I 4.18 & II. I 6 & 9.47 & \\
\hline Solution No... & . (1) & (2) & $(3)$ & (4) & $(5)$ & (6) & (7) & \\
\hline $\begin{array}{c}\text { Dry wt. of gel- } \\
\text { atin discs... }\end{array}$ & 0.340 & 0.341 & 0.341 & 0.342 & 0.342 & 0.345 & 0.345 & \\
\hline Solution...... & $\begin{array}{c}6 \mathrm{cc} . \\
1 \mathrm{M} \\
\text { monosod } \\
\text { citrate } \\
+4 \mathrm{cc} . \\
1 \mathrm{M} \\
\text { disod. } \\
\text { citrate } \\
+90 \mathrm{cc} \\
\mathrm{H}_{2} \mathrm{O}\end{array}$ & $\begin{array}{c}4 \mathrm{cc} . \\
1 M \\
\text { monosod. } \\
\text { citrate } \\
+6 \mathrm{cc} . \\
1 M \\
\text { disod. } \\
\text { citrate } \\
+90 \mathrm{cc} . \\
\mathrm{H}_{2} \mathrm{O}\end{array}$ & $\begin{array}{l}2 \mathrm{cc} \\
1 M \\
\text { monosod } \\
\text { citrate } \\
+8 \mathrm{cc} . \\
1 M \\
\text { disod. } \\
\text { citrate } \\
+90 \mathrm{cc} . \\
\text { H3O } \\
\text { Gain in }\end{array}$ & $\begin{array}{l}10 \mathrm{cc} \\
1 \mathrm{M} \\
1 . \text { disod. } \\
\text { citrate } \\
+90 \mathrm{cc} . \\
\mathrm{H}_{9} \mathrm{O}\end{array}$ & $\begin{array}{c}8 \mathrm{cc} . \\
1 M \\
\text { disod. } \\
\text { citrate } \\
+2 \mathrm{cc} . \\
1 M \\
\text { trisod. } \\
\text { citrate } \\
+90 \mathrm{cc} . \\
\mathrm{H}_{2} \mathrm{O} \\
\text { one part of }\end{array}$ & $\begin{array}{c}6 \mathrm{cc} . \\
1 M \\
\text { disod. } \\
\text { citrate } \\
+4 \mathrm{cc} . \\
1 M \\
\text { trisod. } \\
\text { citrate } \\
+90 \mathrm{cc} . \\
\mathrm{H}_{3} \mathrm{O} \\
\text { gelatin. } \\
\end{array}$ & $\begin{array}{c}4 \mathrm{cc} . \\
1 M \\
\text { disod. } \\
\text { citrate } \\
+6 \mathrm{cc} . \\
1 M \\
\text { trisod. } \\
\text { citrate } \\
+90 \mathrm{cc} \\
\mathrm{H}_{2} \mathrm{O}\end{array}$ & \\
\hline 24 & 7.60 & $7 \cdot 50$ & $7 \cdot 55$ & 8.02 & 8.05 & 8.22 & 8.26 & \\
\hline 48 & $8.6 \mathrm{r}$ & 8.45 & 8.44 & 8.93 & 8.96 & 8.80 & 9.23 & \\
\hline 72 & 9.27 & 9.00 & 9.08 & $9 \cdot 46$ & 9.50 & $9 \cdot 48$ & 10.02 & \\
\hline Solution No... & (8) & (9) & (10) & (II) & (12) & (13) & $\left(I_{4}\right)$ & \\
\hline $\begin{array}{l}\text { Dry wt. of gel- } \\
\text { atin discs... }\end{array}$ & 0.346 & 0.350 & 0.351 & 0.352 & 0.352 & 0.353 & 0.353 & 0.350 \\
\hline Solution...... & $\begin{array}{c}2 \mathrm{cc} . \\
1 M \\
\text { disod. } \\
\text { citrate } \\
+8 \mathrm{cc} . \\
1 M \\
\text { trisod. } \\
\text { citrate } \\
+90 \mathrm{cc} .\end{array}$ & $\begin{array}{c}10 \mathrm{cc} \\
1 \mathrm{M} \\
\text { trisod. } \\
\text { citrate } \\
+90 \mathrm{cc} . \\
\mathrm{H}_{2} \mathrm{O}\end{array}$ & $\begin{array}{c}8 \mathrm{cc} \\
1 \mathrm{M} \\
\text { trisod. } \\
\text { citrate } \\
+2 \mathrm{cc} . \\
1 \mathrm{~N} \\
\mathrm{NaOH} \\
+90 \mathrm{cc} \\
\mathrm{H}_{2} \mathrm{O}\end{array}$ & $\begin{array}{c}6 \mathrm{cc} \\
1 \mathrm{M} \\
\text { trisod. } \\
\text { citrate } \\
+4 \mathrm{cc} \\
1 \mathrm{~N} \\
\mathrm{NaOH} \\
+90 \mathrm{cc} \\
+\mathrm{H}_{2} \mathrm{O}\end{array}$ & $\begin{array}{c}4 \mathrm{cc} . \\
1 \mathrm{M} \\
\text { trisod. } \\
\text { citrate } \\
+6 \mathrm{cc} . \\
1 \mathrm{~N} \\
\mathrm{NaOH} \\
+90 \mathrm{cc} \\
\mathrm{H} \mathrm{O}\end{array}$ & $\begin{array}{c}2 \mathrm{cc} \\
1 M \\
\text { trisod. } \\
\text { citrate } \\
+8 \mathrm{cc} . \\
1 N \\
\mathrm{NaOH} \\
+90 \mathrm{cc} \\
\mathrm{H}_{2} \mathrm{O}\end{array}$ & $\begin{array}{c}10 \mathrm{cc} \\
1 \mathrm{~N} \\
\mathrm{NaOH} \\
+90 \mathrm{cc} . \\
\mathrm{H}_{2} \mathrm{O}\end{array}$ & $\underset{\mathrm{H}_{2} \mathrm{O}}{100 \mathrm{cc}}$ \\
\hline Hours in the & & & Gain in & parts, of & one part of & gelatin. & & \\
\hline 24 & 8.19 & 8.16 & $10.5 \mathrm{I}$ & 14.22 & 17.01 & $24.22+$ & $\begin{array}{c}\text { dis- } \\
\text { solving }\end{array}$ & 6.35 \\
\hline 48 & $9 \cdot 34$ & 9.03 & $\$ 2.90$ & 20.54 & $27.83 t$ & $\begin{array}{l}\text { dis- } \\
\text { solving }\end{array}$ & $\begin{array}{l}\text { dis- } \\
\text { solved }\end{array}$ & $7 \cdot 12$ \\
\hline 72 & 9.54 & $9 \cdot 4^{\mathrm{r}}$ & I 5.06 & $28.83+$ & $\begin{array}{c}\text { dis- } \\
\text { solving }\end{array}$ & & & 8. I I \\
\hline Solution No. . & (15) & (I6) & $(17)$ & (18) & $(19)$ & $(20)$ & $(2 \mathrm{I})$ & $\left(\mathrm{H}_{2} \mathrm{O}\right)$ \\
\hline
\end{tabular}


Between monosodium citrate, disodium citrate and trisodium citrate there is little change in the amount of water absorbed, this portion of the curve describing a gentle arc. As the excess of alkali appears in the mixture, the curve again ascends steeply.

V. Experiments with Carbonate Mixtures.

The swelling of gelatin in carbonate mixtures has also been given some study. The results here again confirm the general conclusions as previously cited for phosphates and citrates. In the carbonate series we began with pure sodium bicarbonate and gradually replaced this by molecularly equivalent amounts of sodium carbonate, and this in turn by of sodium hydroxide. The effects upon the swelling of gelatin as we pass from the sodium bicarbonate through the sodium carbonate to sodium hydroxide is shown in Fig. I4 and Table XIV. The curves show the amounts of swelling at the end of $5 \frac{1}{2}, 29$ and 53 hours. As is clearly apparent, gelatin swells more in a solution of sodium bicarbonate than in pure water and the amount of this swelling increases progressively as we pass from the sodium bicarbonate to sodium carbonate and then more abruptly as we enter the realm of the purer sodium hydroxide.

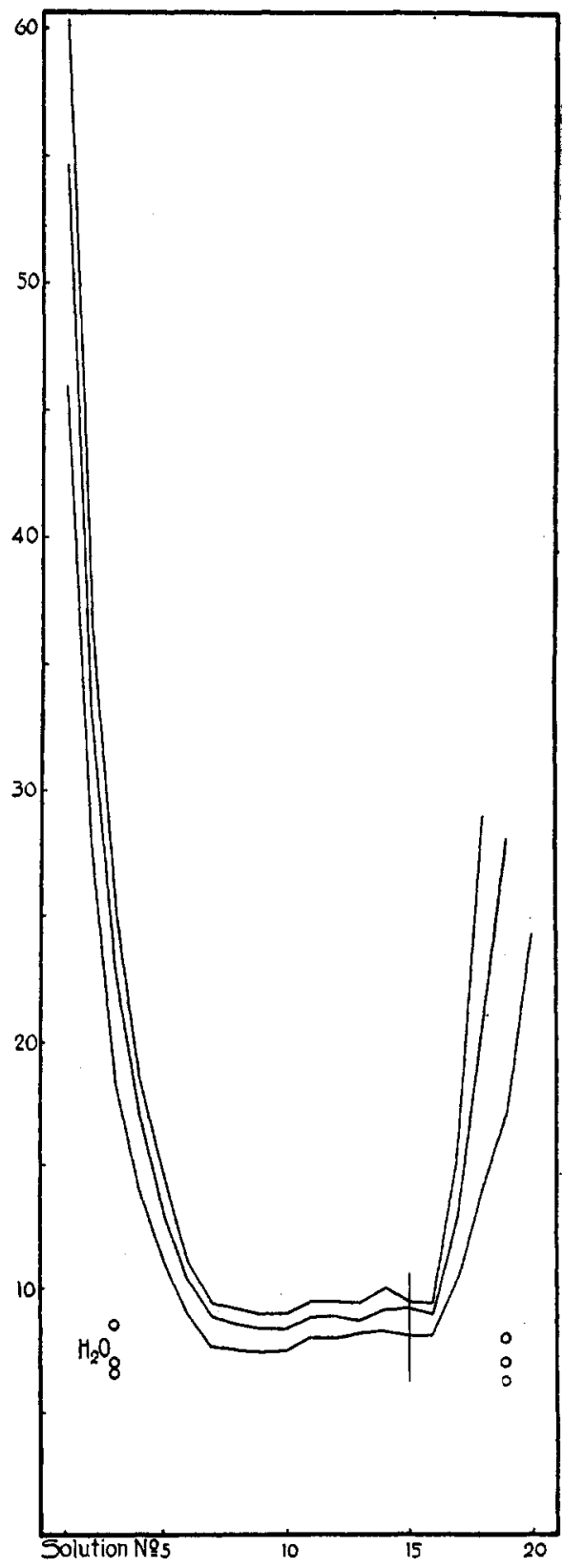

Fig. 13. 
TABLE XIV.

Gelatin-Sodium Bicarbonate, to Sodium Carbonate, to Sodium Hydroxide.

Dry wt. of gel.

atin disc...

Solution.......

Fours in the

$\begin{array}{lllllll}0.368 & 0.369 & 0.370 & 0.370 & 0.371 & 0.371 & 0.372\end{array}$

$2 \mathrm{cc} \quad 1.9 \mathrm{cc} . \quad 1.8 \mathrm{cc} .1 .7 \mathrm{cc} . \quad 1.6 \mathrm{cc} .1 .5 \mathrm{cc} .1 .4 \mathrm{cc}$.

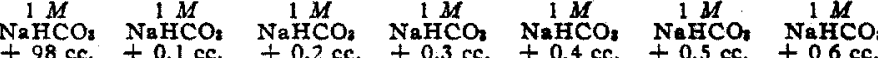

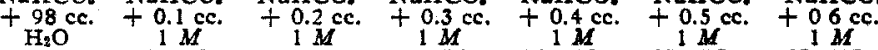

$\mathrm{Na}_{2} \mathrm{CO} \mathrm{Na}_{2} \mathrm{CO}_{2} \mathrm{Na}_{2} \mathrm{CO}_{2} \mathrm{Na}_{2} \mathrm{CO}_{2} \mathrm{Na} \mathrm{CO}_{2} \mathrm{Na}_{2} \mathrm{CO}_{2}$

$+\underset{\mathrm{H}_{2} \mathrm{O}}{9 \mathrm{cc}}+\underset{\mathrm{H}_{2} \mathrm{O}}{9.8 \mathrm{cc}}+\underset{\mathrm{H}_{2} \mathrm{O}}{9 \mathrm{cc}}+\underset{\mathrm{H}_{2} \mathrm{O}}{9 \mathrm{cc}}+\underset{\mathrm{H}_{8} \mathrm{O}}{9 \mathrm{cc}}+\underset{\mathrm{H}_{2} \mathrm{O}}{98}$ Gain in parts, of one part of gelatin.

$\begin{array}{lccccccc}5.5 & 5.18 & 5.30 & 5.65 & 6.00 & 5.66 & 5.58 & 5.97 \\ 29 & \text { II } .52 & 11.84 & 12.07 & 12.56 & 11.91 & 12.00 & 12.35 \\ 53 & 13.05 & 13.40 & 13.51 & 14.00 & 13.30 & 13.33 & 13.75 \\ \text { tion No... } & (1) & (2) & (3) & (4) & (5) & (6) & (7)\end{array}$

Dry wt. of gel-

atin disc....

Solution......

Hours in the

$\begin{array}{lllllll}0.372 & 0.373 & 0.375 & 0.376 & 0.377 & 0.377 & 0.379\end{array}$

$\begin{array}{lllllll}1.3 \mathrm{cc} & 1.2 \mathrm{cc} & 1.1 \mathrm{cc} . & 1.0 \mathrm{cc} . & 0.9 \mathrm{cc} . & 0.8 \mathrm{cc} & 0.7 \mathrm{cc}\end{array}$

$\mathrm{NaHCO}$ NaHCO, NeHCO $\mathrm{NaHCO} \mathrm{NaHCO}_{2} \mathrm{NaHCO} \mathrm{NaHCO}_{2}$

$+0.7 \mathrm{cc}+0.8 \mathrm{cc}+0.9 \mathrm{cc}+1.0 \mathrm{cc}+1.1 \mathrm{cc}+1.2 \mathrm{cc}+1.3 \mathrm{cc}$

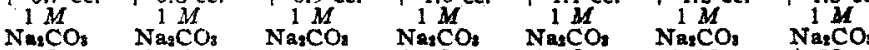

$+98 \mathrm{cc}$. $+98 \mathrm{cc}+98 \mathrm{cc}+98 \mathrm{cc}$. $+98 \mathrm{cc}+98 \mathrm{cc}$. $+98 \mathrm{cc}$ solution.

$\mathrm{H}_{3} \mathrm{O} \mathrm{H}_{3} \mathrm{O}$ Gain in parts, ol one part of gelatin.

$\begin{array}{lccccccc}5.5 & 5.70 & 6.25 & 6.40 & 6.32 & 6.51 & 6.18 & 6.00 \\ 29 & \mathrm{II} .90 & 12.20 & 12.22 & 12.06 & 12.30 & 12.16 & 12.11 \\ 53 & 13.4 \mathrm{I} & 13.47 & 13.60 & 13.55 & 13.71 & 13.63 & 13.50 \\ \text { tion No... } & (8) & (9) & (10) & (1 \mathrm{I}) & (12) & (13) & (14)\end{array}$

Dry wt. of gel-

atin disc....

Solution......

$\begin{array}{lllllll}0.379 & 0.379 & 0.380 & 0.380 & 0.380 & 0.381 & 0.382\end{array}$

$\begin{array}{ccccccc}0.6 \mathrm{cc} & 0.5 \mathrm{cc} & 0.4 \mathrm{cc} & 0.3 \mathrm{cc} & 0.2 \mathrm{cc} . & 0.1 \mathrm{cc} & 2.0 \mathrm{cc} .\end{array}$

$\mathrm{NaHCO}$ NaHCO NaHCO NaHCO NaHCO $\mathrm{NaHCO}_{2} \mathrm{Na}_{2} \mathrm{CO}$

$+1.4 \mathrm{cc}+1.5 \mathrm{cc}+1.6 \mathrm{cc}+1.7 \mathrm{cc}+1.8 \mathrm{cc}+1.9 \mathrm{cc}$

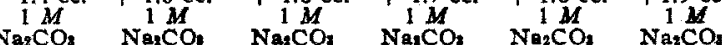

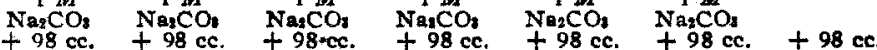

$+\underset{\mathrm{H}_{2} \mathrm{O}}{98 \mathrm{cc}}+\underset{\mathrm{H}_{2} \mathrm{O}}{98}+\underset{\mathrm{H}_{2} \mathrm{O}}{98 \mathrm{cc}}+\underset{\mathrm{H}_{3} \mathrm{O}}{98 \mathrm{cc}}+\underset{\mathrm{H}_{2} \mathrm{O}}{98 \mathrm{cc}}+\underset{\mathrm{H}_{2} \mathrm{O}}{98 \mathrm{cc}}+\underset{\mathrm{H}_{7} \mathrm{O}}{98 \mathrm{cc}}$

Hours in the solution. Gain in parts, of one part of gelatin.

$\begin{array}{lccccccr}5.5 & 6.50 & 6.30 & 6.50 & 6.34 & 6.10 & 6.51 & 6.35 \\ 29 & 12.60 & 12.00 & 12.13 & 12.16 & 12.21 & 12.00 & 12.35 \\ 53 & 14.01 & 13.27 & 13.35 & 13.52 & 13.60 & 13.14 & 13.50 \\ \text { tion No... } & (15) & (16) & (17) & (18) & (19) & (20) & (21)\end{array}$

- Dry wt. of gel-

atin disc.....

Solution......

Fours in the

(15) (I6) (I7)

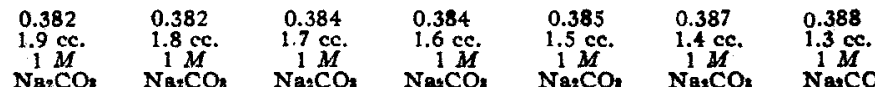

$\mathrm{Na}_{2} \mathrm{CO}_{2} \mathrm{Na}_{2} \mathrm{CO}_{3} \quad \mathrm{Na}_{2} \mathrm{CO}_{3} \mathrm{Na}_{2} \mathrm{CO}_{3} \quad \mathrm{Na}_{2} \mathrm{CO}_{2} \quad \mathrm{Na}_{2} \mathrm{CO}_{2} \quad \mathrm{Na}_{3} \mathrm{MO}$

$+0.1 \mathrm{cc}+0.2 \mathrm{cc}+\mathrm{C}_{1.3 \mathrm{~N}} \mathrm{cc}+0.4 \mathrm{cc}+0.5 \mathrm{cc}+0.6 \mathrm{cc}+0.7 \mathrm{cc}$

$\mathrm{NaOH} \mathrm{NaOH} \mathrm{NaOH} \mathrm{NaOH} \mathrm{NuOH} \mathrm{NaOH} \mathrm{NaOH}$

$+98 \mathrm{cc}$ + $+98 \mathrm{cc}+98 \mathrm{cc}$ + $98 \mathrm{cc}$ + $98 \mathrm{cc}+98 \mathrm{cc}+98 \mathrm{cc}$ solution. Gain in parts, of one part of gelatin.

$\begin{array}{lccccccr}5.5 & 6.92 & 7.21 & 7.45 & 7.40 & 8.20 & 8.78 & 9.80 \\ 29 & 13.01 & 13.28 & 13.09 & 13.78 & 14.65 & 15.20 & 16.34 \\ 53 & 14.26 & 14.42 & 14.24 & 15.02 & 15.63 & 16.13 & 17.24 \\ \text { ion No... } & (22) & (23) & (24) & (25) & (26) & (27) & (28)\end{array}$

Solution No.. .

(22)

(23)

(24)

25)

(26)

$(27)$

(28) 
TABLE XIV (continued).

\begin{tabular}{|c|c|c|c|c|c|c|c|}
\hline $\begin{array}{c}\text { Dry wt. of gel- } \\
\text { atin disc.... }\end{array}$ & 0.390 & 0.390 & 0.390 & 0.392 & 0.393 & 0.393 & 0.395 \\
\hline Solution...... & $\begin{array}{l}1.2 \mathrm{cc} \\
1 \mathrm{M} \\
\mathrm{NaCO} \\
+0.8 \mathrm{cc} . \\
1 \mathrm{~N} \\
\mathrm{NaOH} \\
+98 \mathrm{cc} \\
\mathrm{H}, \mathrm{O}\end{array}$ & $\begin{array}{c}1.1 \mathrm{cc} . \\
1 \mathrm{M} \\
\mathrm{NaCO} \\
+0.9 \mathrm{cc} . \\
1 \mathrm{~N} \\
\mathrm{NaOH} \\
+98 \mathrm{cc} . \\
\mathrm{H}_{2} \mathrm{O}\end{array}$ & $\begin{array}{c}10 \mathrm{cc} . \\
1 \mathrm{M} \\
\mathrm{Na} \mathrm{CO} \\
+1.0 \mathrm{cc} . \\
1 \mathrm{~N} \\
\mathrm{NaOH} \\
+98 \mathrm{cc} \\
\mathrm{H}, \mathrm{O}\end{array}$ & $\begin{array}{l}0.9 \mathrm{cc} . \\
1 \mathrm{M} \\
\mathrm{Na} \mathrm{CO} \\
+1.1 \mathrm{cc} . \\
1 \mathrm{~N} \\
\mathrm{NaOH} \\
+98 \mathrm{cc} . \\
\mathrm{H} 1 \mathrm{O} .\end{array}$ & $\begin{array}{c}0.8 \mathrm{cc} . \\
1 \mathrm{M} \\
\mathrm{Na}_{2} \mathrm{CO} \\
+1.2 \mathrm{cc} \\
1 \mathrm{~N} \\
\mathrm{NaOH} \\
+98 \mathrm{cc} . \\
\mathrm{H}_{2} \mathrm{O} .\end{array}$ & $\begin{array}{c}0.7 \mathrm{cc} . \\
1 \mathrm{M} \\
\mathrm{Na} \mathrm{CO}_{2} \\
+1.3 \mathrm{cc} . \\
1 \mathrm{~N} \\
\mathrm{NaOH} \\
+98 \mathrm{cc} . \\
\mathrm{H}_{2} \mathrm{O}\end{array}$ & $\begin{array}{c}0.6 \mathrm{cc} . \\
1 \mathrm{M} \\
\mathrm{NaCO} \\
+1.4 \mathrm{cc} . \\
1 \mathrm{~N} \\
\mathrm{NaOH} \\
+98 \mathrm{cc} . \\
\mathrm{H}_{2} \mathrm{O}\end{array}$ \\
\hline solution. & & & & & & & \\
\hline $5 \cdot 5$ & 9.58 & 9.93 & 10.18 & 10.14 & II .85 & 11.70 & II. 40 \\
\hline 29 & 16.34 & I $7 \cdot 5$ I & $17 \cdot 55$ & 17.51 & 19.84 & 20.32 & 20.10 \\
\hline $\begin{array}{c}53 \\
\text { Solution No... }\end{array}$ & $\begin{array}{c}17.28 \\
(29)\end{array}$ & $\begin{array}{c}\text { I8.5 I } \\
(30)\end{array}$ & $\begin{array}{c}18.76 \\
(3 I)\end{array}$ & $\begin{array}{c}18.13 \\
(32)\end{array}$ & $\begin{array}{c}20.45 \\
(33)\end{array}$ & $\begin{array}{c}21 \cdot 94 \\
(34)\end{array}$ & $\begin{array}{c}21.76 \\
(35)\end{array}$ \\
\hline $\begin{array}{c}\text { Dry wt. of gel- } \\
\text { atin disc.... }\end{array}$ & 0.395 & 0.396 & 0.396 & 0.396 & 0.397 & 0.397 & 0.368 \\
\hline Solution..... & $\begin{array}{c}0.5 \mathrm{cc} \\
1 \mathrm{M} \\
\mathrm{Na} \mathrm{CO} \\
+1.5 \mathrm{cc} . \\
1 \mathrm{~N} \\
\mathrm{NaOH} \\
+98 \mathrm{cc} . \\
\mathrm{H}_{2} \mathrm{O}\end{array}$ & $\begin{array}{c}0.4 \mathrm{cc} . \\
1 \mathrm{M} \\
\mathrm{NacO} \\
+1.6 \mathrm{cc} . \\
1 N \\
\mathrm{NaOH} \\
+98 \mathrm{cc} . \\
\mathrm{H} \mathrm{O}\end{array}$ & $\begin{array}{c}0.3 \mathrm{cc} . \\
1 \mathrm{M} \\
\mathrm{Na}_{2} \mathrm{CO} \\
+1.7 \mathrm{cc} . \\
1 \mathrm{~N} \\
\mathrm{NaOH} \\
+98 \mathrm{cc} . \\
\mathrm{H} \mathrm{H}_{2} \mathrm{O} \\
\text { in in parts, }\end{array}$ & $\begin{array}{c}0.2 \mathrm{cc} . \\
1 \mathrm{M} \\
\mathrm{Na} \mathrm{CO} \\
+1.8 \mathrm{cc} . \\
1 \mathrm{~N} \\
\mathrm{NaOH} \\
+98 \mathrm{cc} . \\
\mathrm{H}, \mathrm{O} \\
\text { of one part }\end{array}$ & $\begin{array}{c}0.1 \mathrm{cc} . \\
1 \mathrm{M} \\
\mathrm{Na} \mathrm{CO}_{2} \\
+1.9 \mathrm{cc} . \\
1 \mathrm{~N} \\
\mathrm{NaOH} \\
+98 \mathrm{cc} . \\
\mathrm{HzO} . \\
\text { of gelatin. }\end{array}$ & $\begin{array}{c}2.0 \mathrm{cc} \\
1 \mathrm{~N} \\
\mathrm{NaOH} \\
+98 \mathrm{cc} . \\
\mathrm{H}_{3} \mathrm{O}\end{array}$ & $\begin{array}{l}100 \mathrm{cc} \\
\mathrm{H}_{2} \mathrm{O}\end{array}$ \\
\hline $5 \cdot 5$ & II . 86 & II.97 & I 1.77 & I I . 75 & II .76 & I3. I 2 & $3 \cdot 36$ \\
\hline 29 & 20.64 & 20.73 & 20.71 & 21.72 & 22.45 & 24.00 & 6.23 \\
\hline $\begin{array}{l}53 \\
\text { olution No... }\end{array}$ & $\begin{array}{c}22.50 \\
(36)\end{array}$ & $\begin{array}{c}22.67 \\
(37)\end{array}$ & $\begin{array}{c}22.24 \\
(38)\end{array}$ & $\begin{array}{c}24 \cdot 90 \\
(39)\end{array}$ & $\begin{array}{c}25.05 \\
(40)\end{array}$ & $\begin{array}{c}29.00 \\
(4 I)\end{array}$ & $\begin{array}{r}7.18 \\
\left(\mathrm{H}_{2} \mathrm{O}\right)\end{array}$ \\
\hline
\end{tabular}

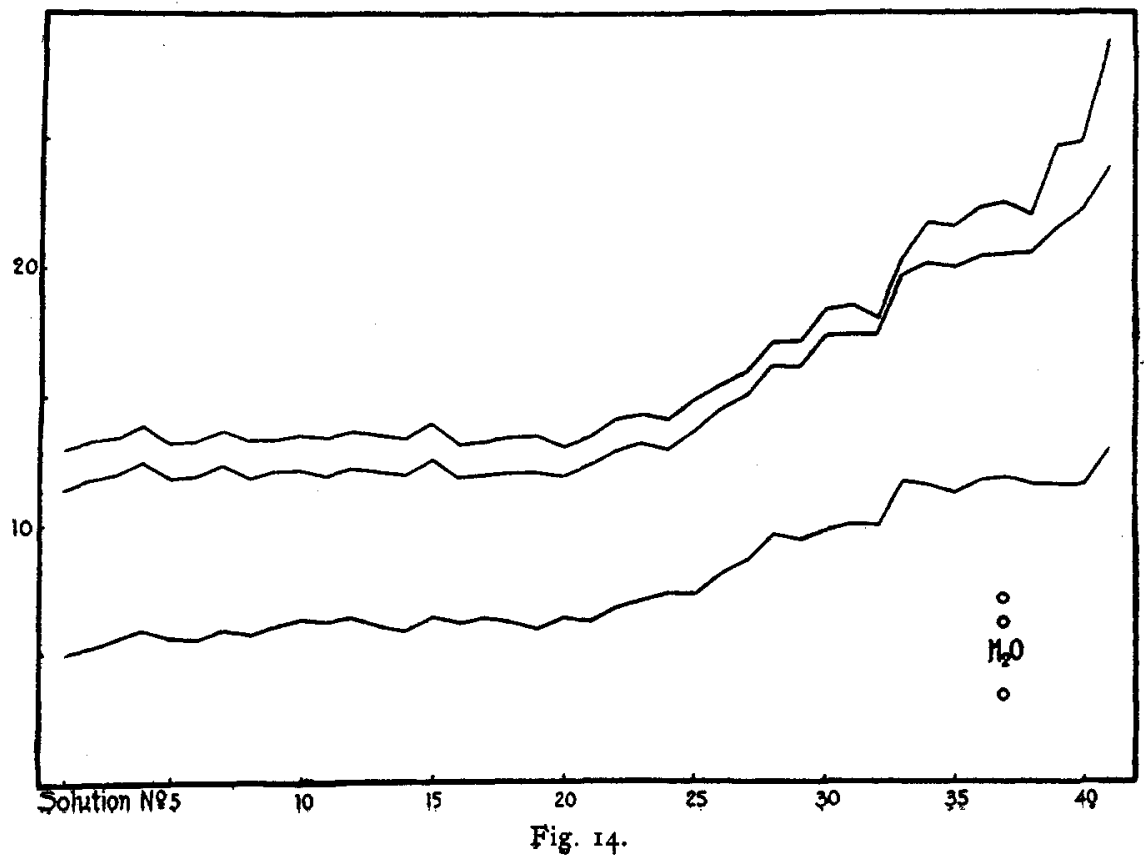




\section{Summary.}

I. Experiments are described showing the amounts of water absorbed by gelatin discs immersed in different concentrations of the primary, binary or ternary salts of phosphoric, citric and carbonic acids. The swelling varies not only with the salt but with its concentration.

2. In further experiments are detailed the absorption of water in pbosphate and citrate mixtures varying from the extreme of the pure acid on the one side through the mono-, di- and trisodium salts of these acids to pure sodium bydroxide on the other. Irrespective of the manner in which these mixtures are prepared (wbether by progressive substitution of one salt for another, through the addition of the requisite acid to an alkali, through the addition of alkali to the proper acid, or tbrough the addition of either acid or alkali to a given salt) it is found, when amount of water absorbed is plotted on the vertical and change in composition of the mixture on the horizontal, that the result yields a V-shaped or U-shaped eurve. From a minimal point in the middle of this curve, there is a progressive increase in water absorption to the left or to the right as acid content or alkali content of the mixture is increased.

3. What has been said of phosphate and citrate mixtures holds also for carbonate mixtures.

4. These findings are held to be applicable to the problem of water absorption by protoplasm and to sustain the old contention that even in the presence of buffer salts there is an increase in water absorption (increased turgor or edema) with every increase in the acid (or alkali) content of the protein colloids found in the involved cell, organ or organism.

Cinctimatr, OHro.

[Contribution from the Eichberg Laboratory of Physiology in the University of Cincinnati.]

\section{ON THE SWELLING OF FIBRIN IN POLYBASIC ACIDS AND THEIR SALTS.}

By Maztin H. Fischer and Martin Benzinger.

Received November 2, 1917.

\section{I.}

It was shown in a previous paper ${ }^{1}$ that water absorption by such a protein as gelatin increases even in the presence of so-called buffer salts with every increase in the acid or alkali content of the mixture on either side of a low point. This conclusion is contrary to much generally accepted opinion. ${ }^{2}$ In order to show that the behavior of gelatin is not exceptional

${ }^{2}$ Martin H. Fischer and Marian O. Hooker, Science, 46, 189 (1917); cf. preceding article.

${ }^{2}$ See Max Koppel, Deut. Arch. klin. Med., I12, 594 (1913); see also Henderson, Palmer and Newburgh, J.Pharm. Exp. Therap., 5, 449 (I914) as well as their numerous followers. 